\title{
Dismantling and rebuilding the trisulfide cofactor demonstrates its essential role in human sulfide quinone oxidoreductase
}

\section{Aaron P. Landry ${ }^{1}$, Sojin Moon ${ }^{1}$, Jenner Bonanata ${ }^{2}$, Uhn Soo Cho ${ }^{1}$, E. Laura Coitiño ${ }^{2}$ and Ruma Banerjee $^{1 *}$}

${ }^{1}$ Department of Biological Chemistry, University of Michigan Medical School, Ann Arbor, MI 48109 and ${ }^{2}$ Laboratorio de Química Teórica y Computacional (LQTC), Instituto de Química Biológica, Facultad de Ciencias and Centro de Investigaciones Biomédicas (CeInBio), Universidad de la República, Iguá 4225, Montevideo 11400, Uruguay

*Address correspondence to: Ruma Banerjee, 4220C MSRB III, 1150 W. Medical Center Dr., University of Michigan, Ann Arbor, MI 48109-0600, Tel: (734) 615-5238; E-mail: rbanerje@umich.edu

\begin{abstract}
Sulfide quinone oxidoreductase (SQR) catalyzes the first step in sulfide clearance, coupling $\mathrm{H}_{2} \mathrm{~S}$ oxidation to coenzyme $Q$ reduction. Recent structures of human SQR revealed a sulfur atom bridging the SQR active site cysteines in a trisulfide configuration. Here, we assessed the importance of this cofactor using kinetic, crystallographic and computational modeling approaches. Cyanolysis of SQR proceeds via formation of an intense charge transfer complex that subsequently decays to eliminate thiocyanate. Cyanolysis leads to reversible loss of SQR activity, which is restored in the presence of sulfide. We captured a crystallographic intermediate in SQR that provides clues as to how the oxidized state of the cysteines is preserved. Computational modeling and MD simulations revealed an $\sim 10^{5}$-fold rate enhancement for nucleophilic addition of sulfide into the trisulfide versus a disulfide cofactor. The cysteine trisulfide in SQR is thus critical for activity and provides a significant catalytic advantage over a cysteine disulfide.
\end{abstract}

\section{Introduction}

Hydrogen sulfide $\left(\mathrm{H}_{2} \mathrm{~S}\right)^{1}$ is a signaling molecule that exerts physiological effects in the cardiovascular, central nervous, and gastrointestinal systems (1-3). $\mathrm{H}_{2} \mathrm{~S}$ is synthesized endogenously in mammals through the activities of cystathionine $\beta$-synthase (4) and cystathionine $\gamma$-lyase (5), as well as 3 -mercaptopyruvate sulfur transferase $(6,7)$. Tissue concentrations of $\mathrm{H}_{2} \mathrm{~S}$ typically range from 10-80 nM (8-10). At higher concentrations, $\mathrm{H}_{2} \mathrm{~S}$ can act as a respiratory poison that blocks the electron transport chain by inhibiting complex IV (11).

Due to the bimodal effects of $\mathrm{H}_{2} \mathrm{~S}$, its levels must be strictly regulated. The accumulation of toxic concentrations of $\mathrm{H}_{2} \mathrm{~S}$ is prevented by its oxidation to thiosulfate and sulfate via the mitochondrial sulfide oxidation pathway (12). The first and committed step in this pathway is catalyzed by sulfide quinone oxidoreductase (SQR), an inner mitochondrial membrane-anchored flavoprotein, which is a member of the flavin disulfide reductase superfamily (13). SQR couples $\mathrm{H}_{2} \mathrm{~S}$ oxidation to coenzyme $\mathrm{Q}_{10}\left(\mathrm{CoQ}_{10}\right)$ reduction $(12,14-16)$. It transfers the oxidized sulfane sulfur to a small molecule acceptor, which is predicted to be glutathione (GSH) under physiological conditions $(15,16)$. Inherited deficiency of SQR presents as Leigh disease (17).

\footnotetext{
${ }^{1}$ Abbreviations used: $\mathrm{H}_{2} \mathrm{~S}$ : hydrogen sulfide, $\mathrm{CoQ}_{10}$ or $\mathrm{CoQ}_{1}$ : coenzyme $\mathrm{Q}_{10}$ or $\mathrm{Q}_{1}, \mathrm{CT}$ : charge transfer, SQR: sulfide quinone oxidoreductase, GSH: glutathione, DTT: dithiothreitol, DHLA: dihydrolipoic acid, HAR: heavy atom reorganization, MD: molecular dynamics, QM/MM: hybrid quantum mechanics/molecular mechanics electronic structure modeling DFT: density functional theory, PCM: polarized continuum model.
} 
While the overall reaction catalyzed by SQRs are similar (18-20), the requirement of a small molecule acceptor by the human enzyme distinguishes it from bacterial homologs, which build long polysulfide chains and can release octasulfur rings as oxidation products (18-20). In contrast, the catalytic cycle of human SQR resembles that of bacterial flavocytochrome $c$ sulfide dehydrogenase, which couples the conversion of sulfide to hydrodisulfide with the reduction of cytochrome $c$ (21). Unexpectedly, the crystal structures of human SQR revealed the presence an additional sulfur bridging the active site cysteines in a trisulfide $(22,23)$. The catalytic relevance of the trisulfide configuration is controversial, and it has been assigned as the inactive (22) or active (23) form of the enzyme. The presence of a cysteine trisulfide in SQR raises questions about how it is built in the active site. To our knowledge, a catalytically relevant cysteine trisulfide would be the first of its kind for a thiol-based redox active cofactor.

A reaction mechanism for human SQR that starts with the trisulfide as the resting form of the enzyme is shown in Fig. 1. The reaction cycle proceeds via two half reactions. In the first half reaction, sulfide adds to the trisulfide at the solvent-accessible Cys-379 to form a ${ }^{379} \mathrm{Cys}-\mathrm{SSH}$ persulfide. The bridging sulfur is retained on ${ }^{201} \mathrm{Cys}_{-} \mathrm{SS}^{-}$persulfide, which forms an unusually intense charge transfer (CT) complex with FAD that is centered at $695 \mathrm{~nm}(14,16,23,24)$. Sulfur transfer from ${ }^{379} \mathrm{Cys}-\mathrm{SSH}$ to small molecule acceptor leads to regeneration of the active site trisulfide with the concomitant two-electron reduction of FAD. In the second half reaction, $\mathrm{FADH}_{2}$ transfers electrons to $\mathrm{CoQ}_{10}$, regenerating the resting enzyme and linking sulfide oxidation to mitochondrial energy metabolism by supplying reduced $\mathrm{CoQ}_{10}$ to Complex III in the electron transport chain (25).

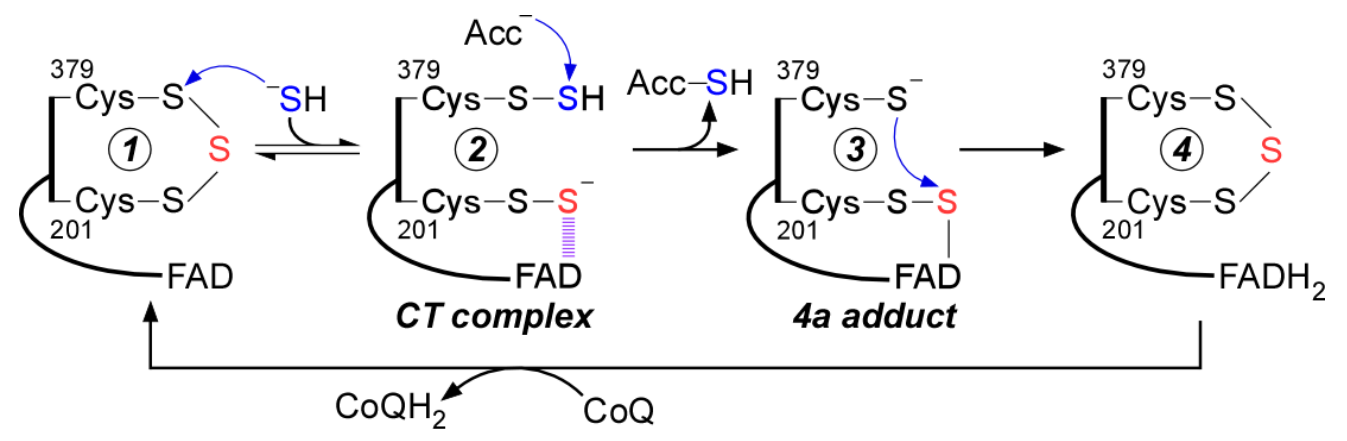

Figure 1. Postulated mechanism for sulfide oxidation catalyzed by human SQR. Proposed mechanism for the reaction catalyzed by SQR. Sulfide adds into the resting cysteine trisulfide (1) to generate a ${ }^{379} \mathrm{Cys}-\mathrm{SSH}$ persulfide and a ${ }^{201} \mathrm{Cys}^{-\mathrm{SS}^{-}}$ persulfide, with the latter participating in a CT complex with FAD (2). Sulfur transfer to a small molecule acceptor proceeds through a putative $4 \mathrm{a}$ adduct (3) to generate the reduced enzyme (4). Electron transfer from $\mathrm{FADH}_{2}$ to $\mathrm{CoQ}$ regenerates the resting enzyme. The oxidized sulfur and bridging sulfur in the cysteine trisulfide are labeled in blue and red, respectively.

In principle, an active site cysteine trisulfide provides several advantages over the conventional disulfide configuration seen in the mechanistically similar flavocytochrome c sulfide dehydrogenase (21). Sulfane sulfur species have increased electrophilic character versus their respective thiols (26), which would enhance the reactivity of the solvent-accessible S $\gamma$ of Cys-379 in SQR towards nucleophilic addition by sulfide. Indeed, the rate of sulfide addition to the cysteine trisulfide of SQR is estimated to be $\sim 2 \times 10^{7}$ fold higher than the rate of sulfide addition to cysteine disulfide in solution $\left(0.6 \mathrm{M}^{-1} \mathrm{~s}^{-1}\right.$ at $\left.\mathrm{pH} 7.4,25{ }^{\circ} \mathrm{C}\right)$ (27). The subsequent formation of persulfide rather than thiolate intermediate on Cys-201 would also enhance its reactivity for facilitating sulfur transfer and electron movement via the putative C4a adduct.

In this study, we report the spectral and kinetic characterization of cyanolysis-induced dismantling followed by sulfide-dependent rebuilding of the trisulfide cofactor. Cyanide treatment destabilized human SQR and led to its inactivation with concomitant loss of the bridging sulfane sulfur. Addition of sulfide to inactive cyanide treated enzyme led to recovery of active SQR, indicating that the oxidation state of the active site cysteines was preserved upon cyanide treatment. Crystallization of SQR with cyanide led to the capture of a ${ }^{379} \mathrm{Cys} N$-( ${ }^{201} \mathrm{Cys}$-disulfanyl)-methanimido thioate intermediate, providing insights into how the trisulfide can be rebuilt following cyanide treatment. Finally, computational modeling indicated that the 
trisulfide configuration provides a significant catalytic advantage over a disulfide in the SQR reaction. Collectively, our study demonstrates that the cysteine trisulfide in SQR is required for its catalytic activity, confers a catalytic edge over a disulfide, and contributes to its structural integrity.

\section{Results}

Formation and decay of the cyanide-induced CT complex in SQR-Mixing SQR with cyanide led to the formation of an intense CT complex characterized by an absorbance maximum at $695 \mathrm{~nm}$ and a shift in the FAD peak from $450 \mathrm{~nm}$ to $420 \mathrm{~nm}$, with isosbestic points at $430 \mathrm{~nm}$ and $505 \mathrm{~nm}$ (Fig. 2A). These spectral features are similar to the CT complexes seen previously with other nucleophiles $(24,28)$. From the dependence of the rate of $\mathrm{CT}$ complex formation on the concentration of cyanide, the following parameters were obtained: $k_{\mathrm{on}}=10,500 \pm 118 \mathrm{M}^{-1} \mathrm{~s}^{-1}, k_{\mathrm{off}}=3.7 \pm 0.6 \mathrm{~s}^{-1}$, and $K_{\mathrm{D}(\mathrm{app})}=348 \pm 52 \mu \mathrm{M}$ at $4{ }^{\circ} \mathrm{C}$ (Fig. $2 \mathrm{~B}, \mathrm{C}$ ). The CT complex is an intermediate in the catalytic cycle of SQR, and sulfide addition to the CT intermediates formed by alternative nucleophiles leads to their decay with the concomitant reduction of FAD $(24,28)$. Similarly, addition of sulfide immediately following cyanide-induced CT complex formation led to its decay with concurrent reduction of FAD (Fig. 2D). This result indicates that the CT complex formed in the presence of cyanide can participate in the first half reaction leading to $\mathrm{FADH}_{2}$ formation.

Decay of the cyanide-induced CT complex and cyanolysis of the cysteine trisulfide-Extended incubation of the cyanide-induced CT complex in the presence of excess cyanide led to its slow decay (Fig. $3 \mathrm{~A}$ ). A $k_{\text {obs }}$ of $0.15 \pm 0.02 \mathrm{~min}^{-1}$ at $20^{\circ} \mathrm{C}$ was observed for the decay of the CT complex in the presence of 5-10 mM KCN (Fig. 3B). The FAD spectrum following CT decay was slightly altered from that in native SQR. Thus, a blue shift in the absorbance maximum from $450 \mathrm{~nm}$ to $447 \mathrm{~nm}$ and a narrowing of the 380 $\mathrm{nm}$ absorption peak were seen (Fig. 3A). The altered spectral features were observed even after the enzyme was desalted to remove excess cyanide, suggesting a change in the flavin electronic environment. Cyanolysis yields $\sim 1 \mathrm{~mol}$ of sulfane sulfur per mol SQR monomer (23), indicating that the native trisulfide state is lost upon prolonged cyanide treatment. To confirm this conclusion, we added sulfite, which forms a strong CT complex when added to native SQR (23), However, sulfite did not elicit spectral changes in cyanide pre-treated and desalted SQR (Fig. 3C).

Sulfide-mediated regeneration of the active site trisulfide-We next assessed the impact of cyanide treatment on SQR activity under steady state turnover conditions. Surprisingly, the specific activity of SQR in the standard assay was similar for the cyanide pre-treated $\left(369 \pm 25 \mu \mathrm{mol} \mathrm{min} \mathrm{mg}^{-1}\right)$ and native $(360 \pm$ $12 \mu \mathrm{mol} \mathrm{min} \mathrm{mg}^{-1}$ ) enzymes. This result suggested that cyanide treated enzyme could be reactivated by rebuilding the trisulfide following cyanolysis of SQR.

We therefore monitored the rate at which the trisulfide is rebuilt using as a measure of the active enzyme, formation of the sulfide-induced CT complex $(14,24)$ (Fig. 4A). For this, the kinetics of CT complex formation was assessed following rapid mixing of sulfide with cyanide pre-treated SQR. Compared to native SQR $\left(k_{\mathrm{obs}}=19.4 \pm 1.8 \mathrm{~s}^{-1}\right)$, CT complex formation was $\sim 12$-fold slower with cyanide pre-treated SQR $\left(1.6 \pm 0.2 \mathrm{~s}^{-1}\right)$ (Fig. 4B). The lag in the absorbance increase at $675 \mathrm{~nm}$ indicated that trisulfide rebuilding limits the rate of CT complex formation in cyanide pre-treated SQR. Consistent with this postulate, incubation of cyanide pre-treated SQR with sulfide for $1 \mathrm{~h}$ at $4{ }^{\circ} \mathrm{C}$ led to FAD reduction (Fig. $4 \mathrm{C}$ ), signaling reformation of the active trisulfide-containing SQR under these conditions. The presence of excess sulfide, which serves as both the sulfur donor and acceptor, led to $\mathrm{FADH}_{2}$ accumulation in the absence of $\mathrm{CoQ}_{1}(14,24)$. The $447 \mathrm{~nm}$ FAD absorption peak observed in cyanide pre-treated SQR (Fig. 2A) shifted to $450 \mathrm{~nm}$ following incubation with sulfide (Fig. 4D), indicating recovery of the native FAD microenvironment. Furthermore, addition of sulfite to regenerated SQR resulted in the formation of a robust CT complex, confirming the presence of a trisulfide in the active site (Fig. 4D). 
A
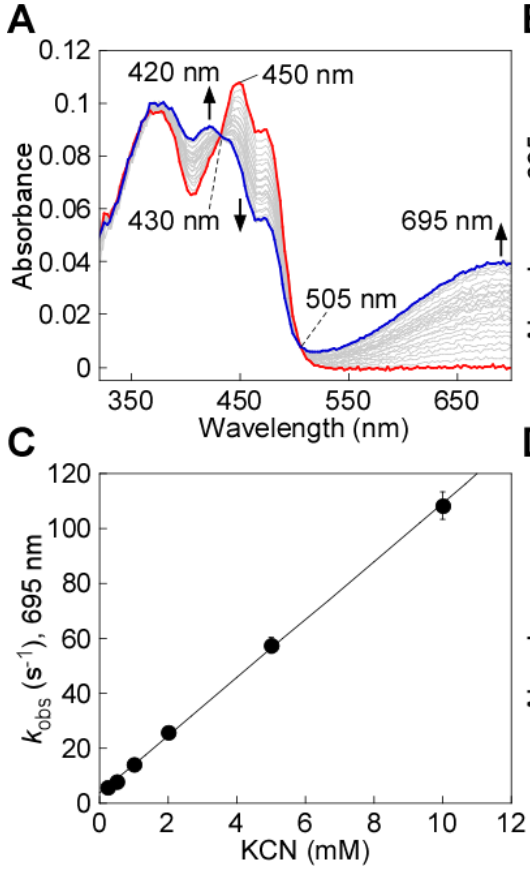

B

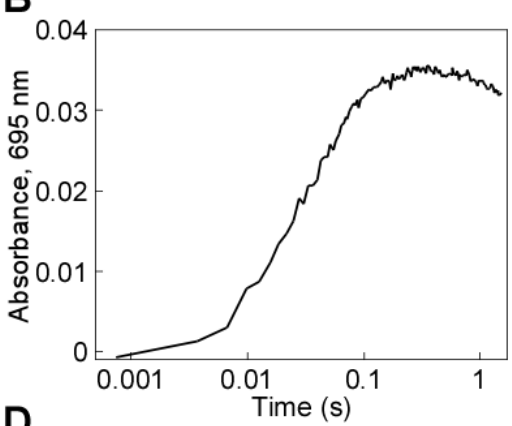

D

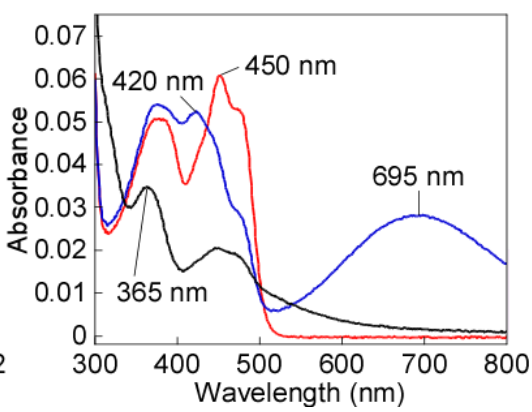

Figure 2. Cyanide-induced CT complex formation in SQR. A, SQR (10 $\mu \mathrm{M}$, red line) in $100 \mathrm{mM}$ potassium phosphate, pH 7.4 containing $0.03 \%$ DHPC, was mixed $1: 1(\mathrm{v} / \mathrm{v})$ with $\mathrm{KCN}(4 \mathrm{mM})$ and monitored over $1.5 \mathrm{~s}$ at $4{ }^{\circ} \mathrm{C}$ for the formation of the cyanideinduced CT complex at $695 \mathrm{~nm}$ (blue line). B, Representative stopped flow kinetic trace for the reaction in (A) monitored at 695 $\mathrm{nm}$. C, Dependence of the $k_{\mathrm{obs}}$ at $4{ }^{\circ} \mathrm{C}$ for cyanide-induced CT complex formation on cyanide concentration. The data are representative of two independent experiments, with each data point obtained in triplicate. $\mathbf{D}, \mathrm{SQR}(5 \mu \mathrm{M}$, red line) was treated with $\mathrm{KCN}(5 \mathrm{mM})$ to form the CT complex (blue line), immediately followed by the addition of $\mathrm{Na}_{2} \mathrm{~S}(200 \mu \mathrm{M})$ and incubated for 5 min at $20^{\circ} \mathrm{C}$, which led to CT complex decay and FAD reduction (black line). The data are representative of three independent experiments.

Cyanolysis of the bridging sulfur decreases SQR protein stability-We consistently observed that cyanide treatment led to an increased tendency for SQR to aggregate at temperatures above $20{ }^{\circ} \mathrm{C}$, indicating that loss of the bridging sulfur in the active site trisulfide leads to protein instability. We therefore investigated the thermal stability of SQR with and without cyanide pre-treatment. Native SQR exhibited a $\mathrm{T}_{\text {agg }}$ of $64.8^{\circ} \mathrm{C}$, compared to $36.5^{\circ} \mathrm{C}$ for cyanide pre-treated enzyme (Fig. 5). Incubation of cyanide pretreated SQR in the presence of excess sulfide increased its stability $\left(\mathrm{T}_{\text {agg }}\right.$ of $\left.56.6{ }^{\circ} \mathrm{C}\right)$. Thus, the decrease in thermal stability of SQR upon loss of the bridging sulfur was largely reversed upon regeneration of the cysteine trisulfide.

Dithiol-mediated reduction of FAD in SQR-As an alternative to cyanolysis, we attempted to extract the bridging sulfur from the SQR trisulfide using DTT, which in principle could reduce the trisulfide to generate free thiols on Cys-379 and Cys-201. Unexpectedly, treatment with DTT led to bleaching of the yellow color associated with SQR (Fig. 6A). Given the known substrate promiscuity of SQR $(24,28)$, we postulate that DTT adds to the resting trisulfide, forming a mixed disulfide and a CT complex. In the second step, an intramolecular displacement by the second thiol in the ${ }^{379}$ Cys-S-S-DTT adduct leads to elimination of oxidized DTT, reduction of FAD, and regeneration of the trisulfide (Fig. 6B).

To further test this model, the reaction of DTT with SQR was characterized by stopped-flow spectroscopy. Upon mixing SQR rapidly with DTT, FADH 2 formation $\left(k_{\text {obs }}=0.36 \pm 0.04 \mathrm{~s}^{-1}\right)$ was observed without accumulation of a CT complex intermediate (Fig. 6A). This contrasted with the reaction of other nucleophiles with SQR, and suggested that resolution of the mixed disulfide, via an intramolecular reaction, 
A
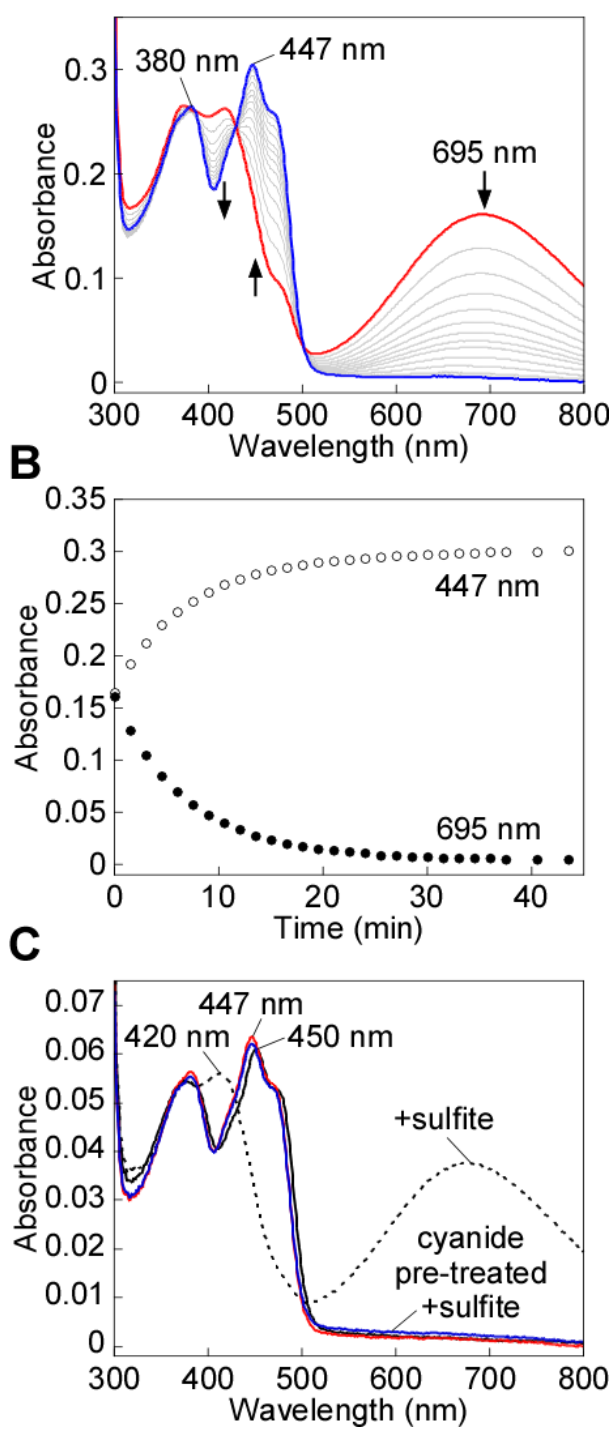

Figure 3. Cyanide-induced CT complex decay in SQR. A, SQR $(25 \mu \mathrm{M})$ in Buffer A was treated with $\mathrm{KCN}(10 \mathrm{mM})$ to form the CT complex (red line), which was monitored over $43 \mathrm{~min}$ at $20^{\circ} \mathrm{C}$ for the complete decay of the CT complex (blue line). B, Kinetic traces for the decay of the cyanide-induced CT complex in (A), monitored at $450 \mathrm{~nm}$ (open circles) and $695 \mathrm{~nm}$ (closed circles). C, SQR ( $5 \mu \mathrm{M}$, solid black line) was treated with sodium sulfite $(5 \mathrm{mM})$ and incubated for 1 min at $20^{\circ} \mathrm{C}$ to form the $\mathrm{CT}$ complex formation (dashed black line). In tandem, SQR $(5 \mu \mathrm{M})$ pre-treated with $\mathrm{KCN}(10 \mathrm{mM})$ and desalted (solid red line) was then treated with sodium sulfite $\left(5 \mathrm{mM}\right.$ ). CT complex formation was not observed after incubation for $1 \mathrm{~min}$ at $20{ }^{\circ} \mathrm{C}$ (solid blue line). The data are representative of three independent experiments.

is more rapid than its formation (Fig. 6B). Next, we tested whether the intact cysteine trisulfide in SQR is required for FAD reduction by DTT. Pre-treatment of SQR with cyanide prevented FAD reduction by DTT (Fig. 6C).

In contrast to DTT, the monothiol, $\beta$-mercaptoethanol, was unable to drive FAD reduction, and formed a stable CT complex instead (Fig. 6C). Like DTT, the dithiol dihydrolipoic acid (DHLA), a physiological reductant, also led to FAD reduction, but only when the cysteine trisulfide was intact (Fig. 6D). 
A
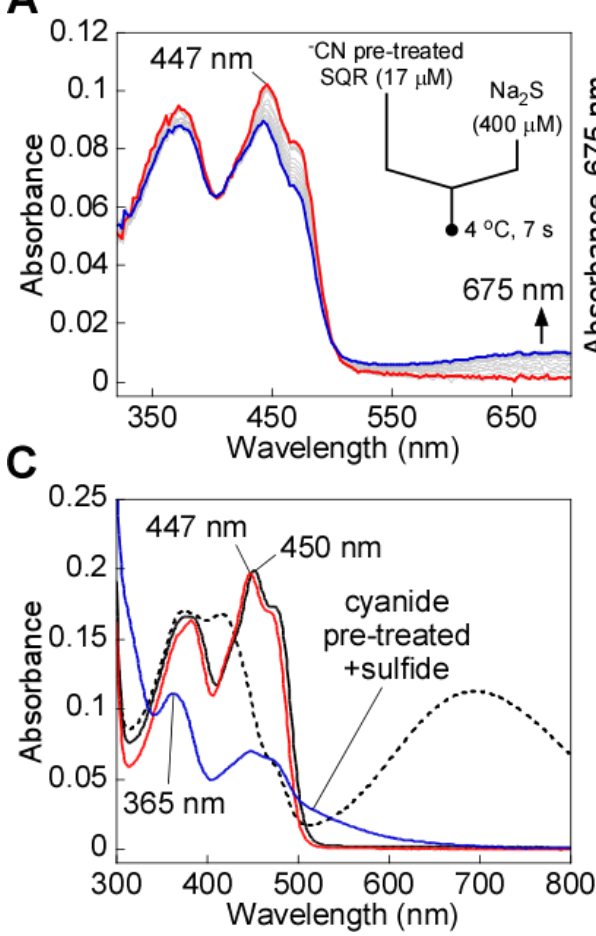

B

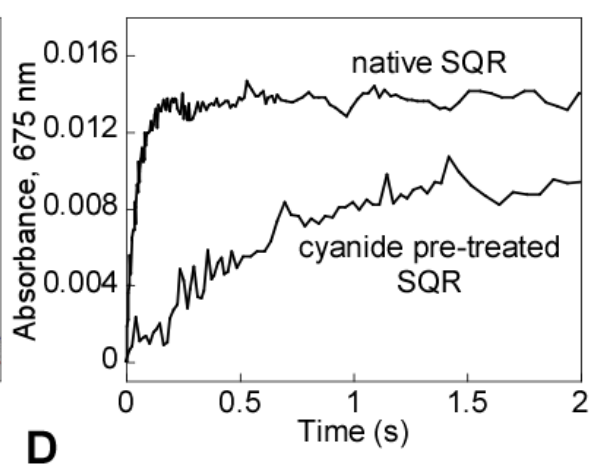

D.07

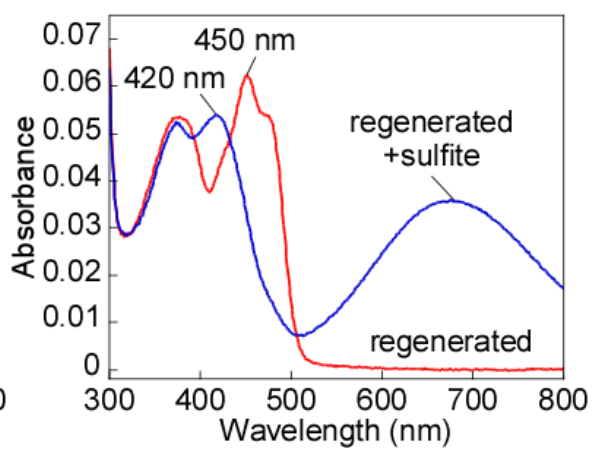

Figure 4. Regeneration of cyanide pre-treated SQR by sulfide. A, Cyanide pre-treated SQR (17 $\mu$ M, red line) in Buffer A was rapidly mixed $1: 1(\mathrm{v} / \mathrm{v})$ with $\mathrm{Na}_{2} \mathrm{~S}(400 \mu \mathrm{M})$ and monitored over a period of $7 \mathrm{~s}$ at $4{ }^{\circ} \mathrm{C}$ for formation of the sulfide-induced $\mathrm{CT}$ complex (blue line). B, Comparison of the kinetic traces at $675 \mathrm{~nm}$ for cyanide pre-treated SQR, as shown in A, versus native SQR mixed with $\mathrm{Na}_{2} \mathrm{~S}(400 \mu \mathrm{M})$ under the same conditions. C, SQR $(17 \mu \mathrm{M}$, solid black line) in Buffer A was treated with KCN (10 $\mathrm{mM}$ ) to form the CT complex (dashed black line) and monitored over $40 \mathrm{~min}$ at $20^{\circ} \mathrm{C}$ for the complete decay of the CT complex and desalted to remove excess cyanide (red line). Cyanide pre-treated SQR was then incubated with $\mathrm{Na}_{2} \mathrm{~S}(300 \mu \mathrm{M})$ for $1 \mathrm{~h}$ at 4 ${ }^{\circ} \mathrm{C}$, which led to FAD reduction (blue trace). D, Cyanide pre-treated SQR, pre-incubated with sulfide under the same conditions as (A) and desalted $(5 \mu \mathrm{M}$, red line), was treated with sulfite $(5 \mathrm{mM})$ and incubated for $1 \mathrm{~min}$ to form the sulfite-induced CT complex (blue line). The data are representative of three independent experiments.

190

191

192

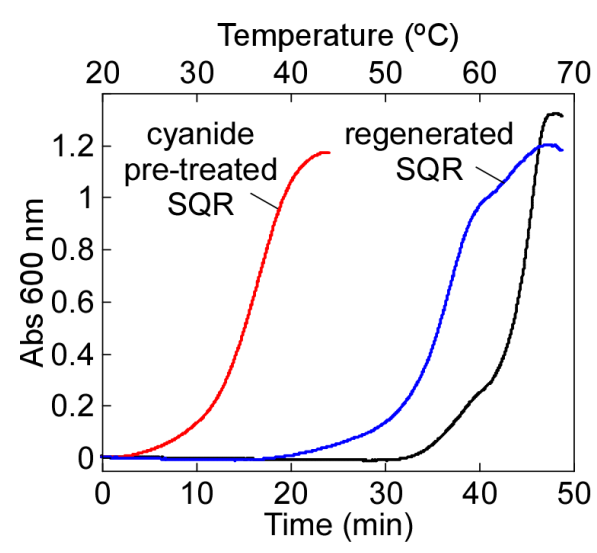

Figure 5. Effect of bridging sulfur extraction on SQR protein stability. SQR $(20 \mu \mathrm{M})$ in Buffer A was pre-treated with KCN $(10 \mathrm{mM})$ for $45 \mathrm{~min}$ at $20^{\circ} \mathrm{C}$ and desalted, followed by incubation with $\mathrm{Na}_{2} \mathrm{~S}(300 \mu \mathrm{M})$ for $1 \mathrm{~h}$ at $4{ }^{\circ} \mathrm{C}$ and a second desalting. A final SQR concentration of $5 \mu \mathrm{M}$ was used for the thermal denaturation assays. The stability of native SQR (black line) versus cyanide pre-treated SQR before (red line) and after (blue line) incubation with sulfide was monitored by the increase in absorbance at $600 \mathrm{~nm}$. The data are representative of three independent experiments. 
A
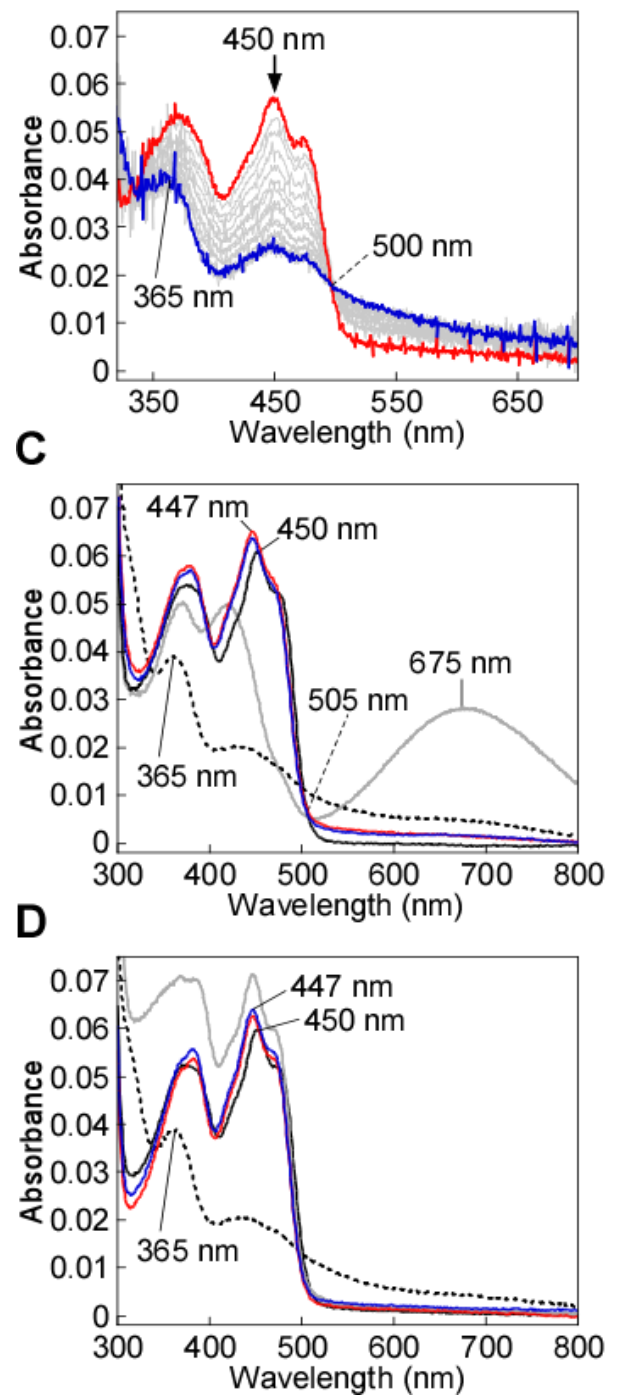

B
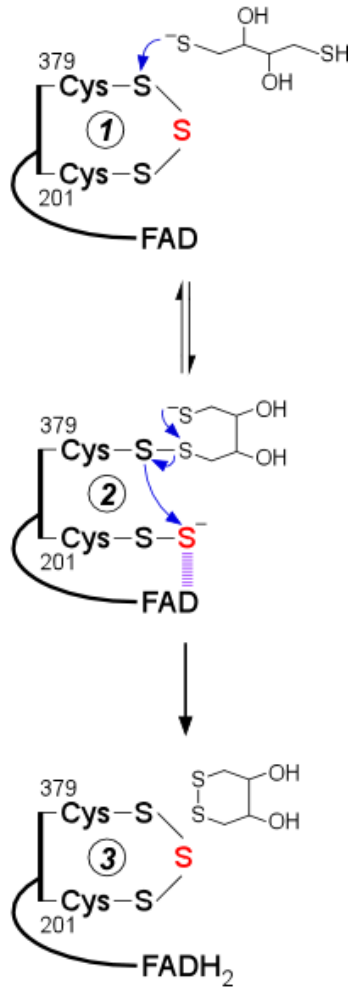

Figure 6. Dithiol-mediated reduction of FAD in SQR. A, SQR $(10 \mu \mathrm{M})$ in Buffer A was rapidly mixed 1:1 (v/v) with DTT (400 $\mu \mathrm{M})$ and FAD reduction was monitored over $7 \mathrm{~s}$ at $4{ }^{\circ} \mathrm{C}$. B, Proposed mechanism for the addition of DTT into the SQR cysteine trisulfide, leading to FAD reduction. DTT adds into the cysteine trisulfide at the solvent-accessible Cys-379 to generate a mixed disulfide and ${ }^{201} \mathrm{Cys}-\mathrm{SS}-$. An intramolecular thiol-disulfide exchange then regenerates the SQR cysteine trisulfide, with electrons moving into FAD. C, SQR $(5 \mu \mathrm{M})$ in Buffer A (solid black line), was treated with DTT $(200 \mu \mathrm{M})$, leading to FAD reduction (dashed black line), or $\beta$-mercaptoethanol $(200 \mu \mathrm{M})$, leading to stable CT complex formation (solid gray line). FAD reduction was not observed in cyanide pre-treated SQR $(5 \mu \mathrm{M}$, solid red line) upon treatment with DTT ( $200 \mu \mathrm{M}$, solid blue line). D, SQR $(5 \mu \mathrm{M})$ under the same conditions as in (C) (solid black line), was treated with DHLA (200 $\mu \mathrm{M})$, leading to FAD reduction (dashed black line), followed by re-oxidation by addition of $\mathrm{CoQ}_{1}(180 \mu \mathrm{M}$, solid gray line). FAD reduction was not observed in cyanide pretreated SQR (5 $\mu \mathrm{M}$, solid red line) upon treatment with DHLA $(200 \mu \mathrm{M}$, solid blue line). The data are representative of three independent experiments.

Structure of $S Q R-C o Q_{1}$ soaked with cyanide-To obtain structural insights into the interaction of cyanide with SQR, crystals of human SQR-CoQ ${ }_{1}$ were soaked with cyanide. The $2.25 \AA$ resolution structure was obtained by molecular replacement using coordinates for the SQR-CoQ ${ }_{1}$ structure (PDB ID: 6OIB) (Table 1). The overall structure (Fig. 7A) is similar to that reported previously for SQR-CoQ ${ }_{1}(23)$. 
Table 1. Crystallographic data collection and refinement statistics*

\begin{tabular}{ll}
\hline & SQR-CoQ $\mathbf{1}_{1}$ with cyanide \\
\hline Space group & $\mathrm{P} 2_{1} 2_{1} 2_{1}$ \\
Unit cell parameters $(\AA)$ & $\mathrm{a}=78.39$ \\
& $\mathrm{~b}=111.75$ \\
$\mathrm{c}=134.05$ \\
$\alpha=\beta=\gamma=90^{\circ}$ \\
Wavelength $(\AA)$ & 1.12723 \\
Data collection statistics & \\
Resolution range $(\AA)$ & $50.00-2.25(2.29-2.25)$ \\
Number of unique reflections & $57050(2696)$ \\
Completeness $(\%)$ & $99.7(96.0)$ \\
$\mathrm{R}_{\text {merge }}$ & $0.175(0.974)$ \\
$\mathrm{R}_{\text {pim }}$ & $0.059(0.359)$ \\
Redundancy & $8.6(6.6)$ \\
Mean $\mathrm{I} / \sigma$ & $14.0(2.3)$ \\
Refinement statistics & \\
Resolution range $(\AA)$ & $39.20-2.24$ \\
$\mathrm{R}_{\text {work }} / \mathrm{R}_{\text {free }}(\%)$ & $17.24 / 21.60$ \\
RMSD bonds $(\AA)$ & 0.008 \\
RMSD angles $($ deg) & 0.922 \\
Average B factor $\left(\AA^{2}\right)$ & 36.62 \\
Number of water molecules & 125 \\
Ramachandran & 96.51 \\
favored $(\%)$ & 3.49 \\
allowed $(\%)$ & 0 \\
not allowed $(\%)$ &
\end{tabular}

*Values in parentheses are for highest-resolution shell.

Surprisingly, strong and continuous electron density was observed between Cys-201 and Cys-379 (Fig. 7B,C). The electron density in the presence of cyanide was more extended than for the trisulfide in the native SQR-CoQ ${ }_{1}+$ sulfide structure (Fig. 7D). We interpret the additional electron density as evidence for the insertion of a cyanide molecule in the trisulfide bridge, forming an $N-\left({ }^{201} \mathrm{Cys}\right.$-disulfanyl)-methanimido thioate intermediate, ${ }^{201} \mathrm{Cys}-\mathrm{S}-\mathrm{S}-\mathrm{N}=\mathrm{CH}-\mathrm{S}-{ }^{379} \mathrm{Cys}$. The relevance of this species to the spectral intermediates observed in the presence of cyanide is discussed later.

$M D$ simulations and $Q M / M M$ reactivity predictors for a disulfide versus trisulfide cofactor-Direct comparison of the active site architecture in representative SQR structures was extracted from $600 \mathrm{~ns}$ trajectories (RMSDs shown in Fig. S1). The simulations provided insights into the structural and dynamical differences between the trisulfide versus a modeled disulfide state, and a chemical rationale for the use of the trisulfide cofactor by SQR. In the disulfide structure (Fig. 8A, right) the sulfur atoms of Cys-201 and Cys-379 are buried (Fig. S2, right), and not in contact with solvent molecules. The S $\gamma$ atoms in the two cysteines exhibit differences in their distance to the C4a in FAD, with Cys-379 being closer (Fig. 8B, right) at distances of $3.4 \AA$ versus $3.9 \AA$ for Cys-201 in the representative structure. These data argue against a catalytic disulfide configuration in SQR, which is reinforced by considerations of the intrinsic electrophilicity of the sulfur atoms calculated at the QM/MM level. 

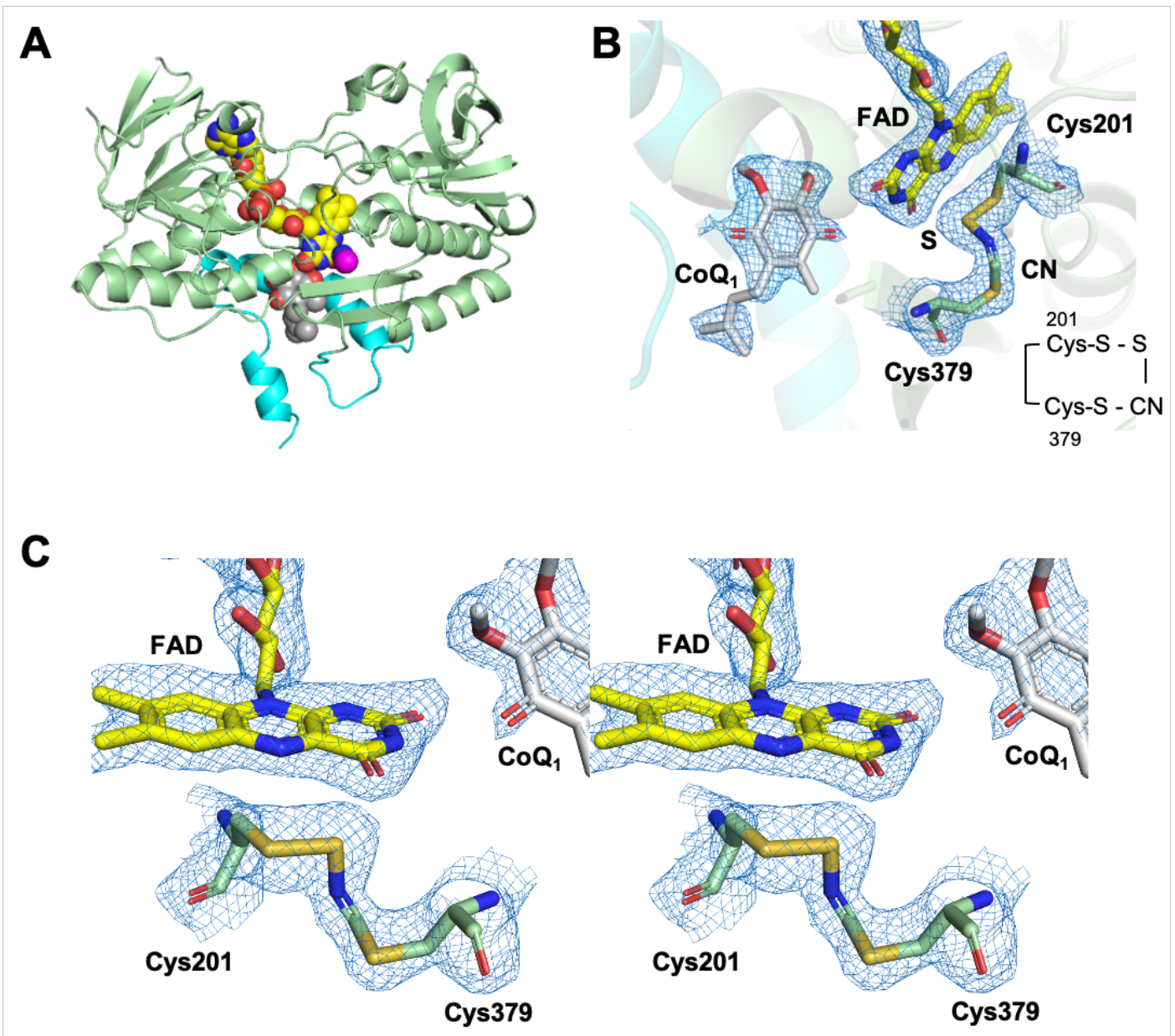

D
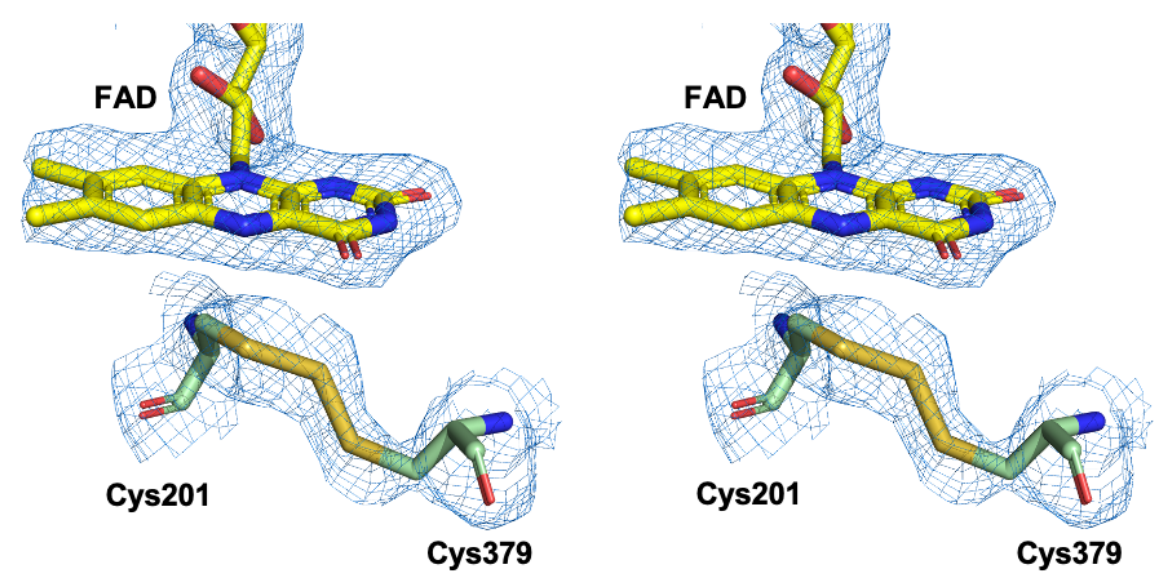

Figure 7. Structure and active site of $S Q R-C o Q_{1}+$ cyanide. A, The overall structure of $S Q R-C o Q_{1}+$ cyanide is shown with $\mathrm{FAD}, \mathrm{CoQ}_{1}$, and cyanide in yellow, grey, and magenta spheres, respectively. The C-terminal membrane-anchoring helices are highlighted in cyan. B, Electron density maps $\left(2 \mathrm{~F}_{\mathrm{o}}-\mathrm{F}_{\mathrm{c}}\right)$ of the active site shown in mesh contoured at $1.0 \sigma$. Cys-201, Cys-379, $\mathrm{FAD}, \mathrm{CoQ}_{1}$, sulfur derived from the trisulfide, and cyanide are shown in stick display. C, Stereo image of the active site of SQR$\mathrm{CoQ}_{1}$ treated with cyanide. The electron densities $\left(2 \mathrm{~F}_{\mathrm{o}}-\mathrm{F}_{\mathrm{c}}\right)$ are contoured at $1.0 \sigma$. D, Stereo image of the active site in SQR-CoQ treated with sulfide (PDB ID: 6OI6) showing the resting trisulfide. Chain A is shown in this figure. 
Trisulfide
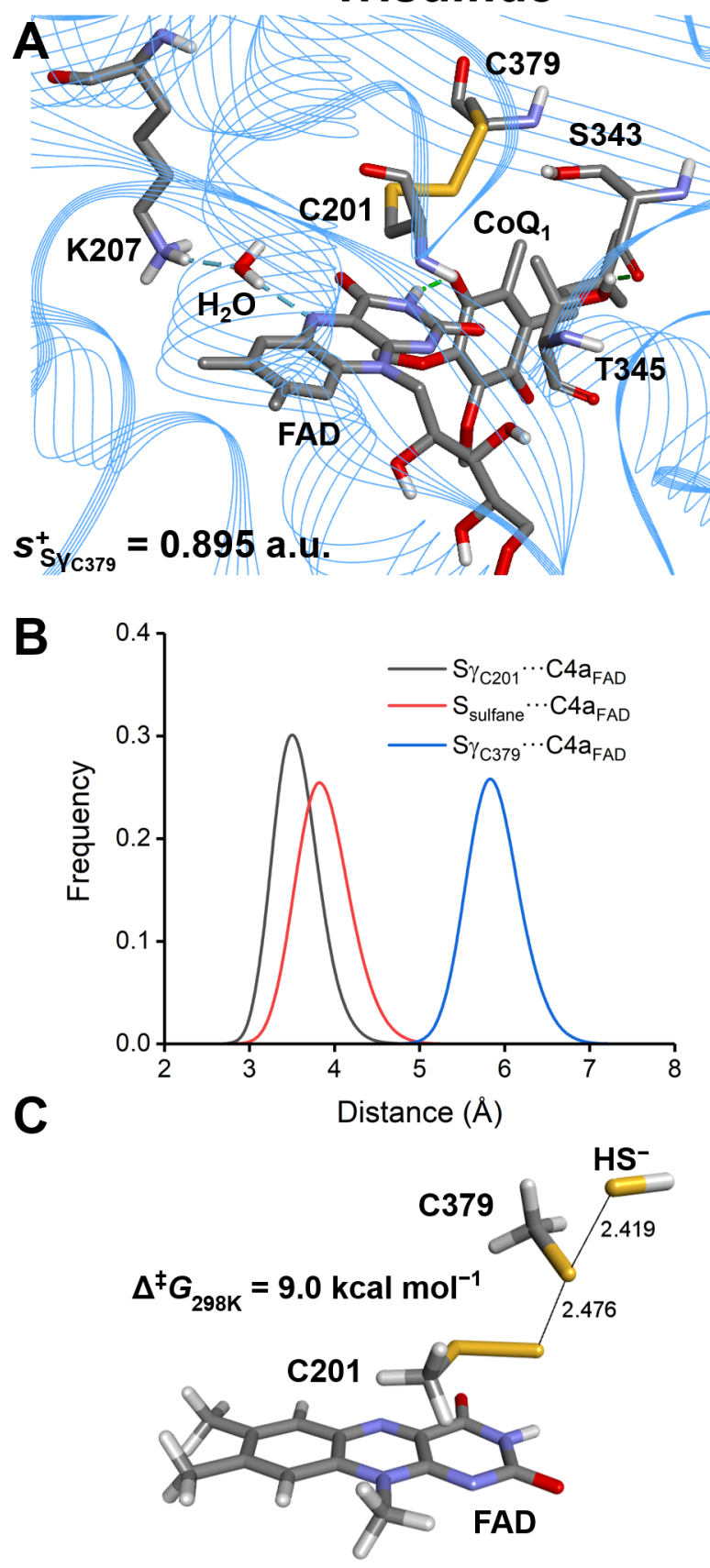

Disulfide
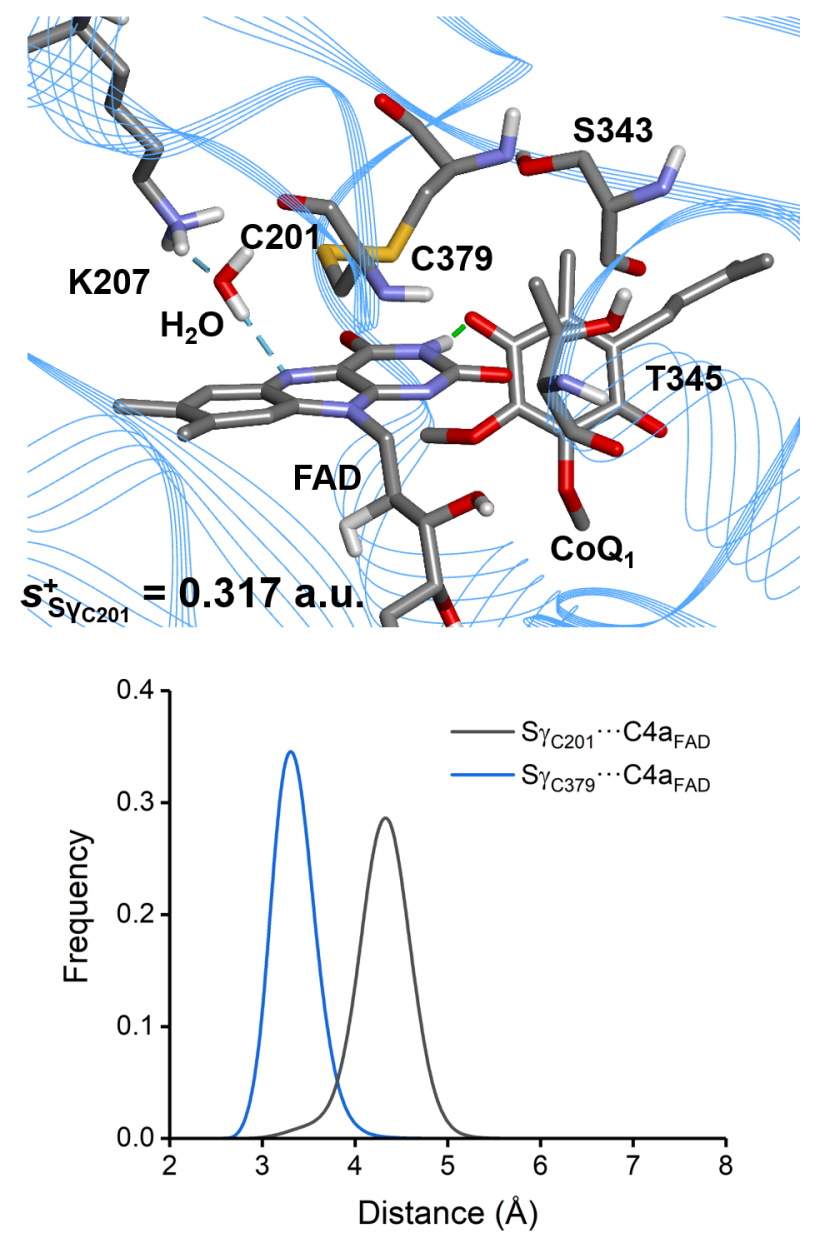

$\Delta^{\ddagger} G_{298 \mathrm{~K}}=15.3 \mathrm{kcal} \mathrm{mol}^{-1}$

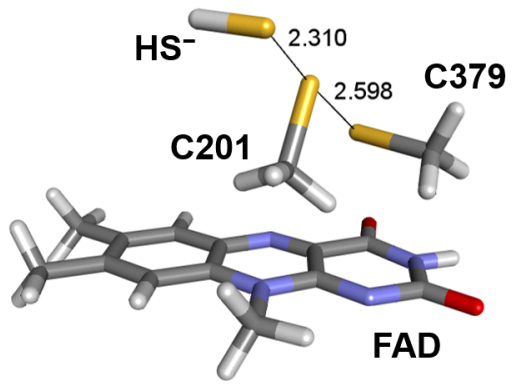

Figure 8. MD simulations and computational modeling of SQR. A, Active site architecture in representative structures corresponding to the most populated cluster from $600 \mathrm{~ns}$ MD simulations of SQR in the trisulfide (left) or disulfide (right) state. Condensed local softness for the most electrophilic S $\gamma$ atom between Cys-201 and Cys-379 is reported for each system in atomic units (a.u.). B, Sulfur-to-C4a FAD distances for $\mathrm{S} \gamma_{\mathrm{C} 201}-\mathrm{C} 4 \mathrm{a}_{\mathrm{FAD}} / \mathrm{S}_{\text {sulfane }}-\mathrm{C} 4 \mathrm{a}_{\mathrm{FAD}} / \mathrm{S} \gamma_{\mathrm{C} 379}-\mathrm{C} 4 \mathrm{a}_{\mathrm{FAD}}$ (trisulfide, left) and $\mathrm{S} \gamma_{\mathrm{C} 201}-\mathrm{C} 4 \mathrm{a}_{\mathrm{FAD}} / \mathrm{S} \gamma_{\mathrm{C} 379}-\mathrm{C} 4 \mathrm{a}_{\mathrm{FAD}}$ (disulfide, right) monitored along the corresponding MD trajectories. $\mathbf{C}$, Structure of the transition states (TS) located for the sulfide anion attack on the trisulfide (left) or disulfide (right) using a reduced model of the active site of SQR at the IEFPCM-DFT level of theory in a dielectric of $\varepsilon=10.125$. Data correspond to interatomic distances in $\AA$ and Gibbs free-energy associated barriers at $298 \mathrm{~K}_{\text {in }} \mathrm{kcal} \mathrm{mol}^{-1}$. 
In the trisulfide model, the $\mathrm{S} \gamma$ atom of Cys-201 is estimated to be intrinsically less electrophilic than Cys-379 $\left(\mathrm{s}^{+}=0.317\right.$ versus 0.895 atomic units). These results support the proposed attack of the sulfide anion on the $\mathrm{S} \gamma$ atom of Cys-379 as the first step in the catalytic mechanism (Fig. 1). In the trisulfide structure (Fig. 8A, left) the sulfur atom of Cys-379 is located in a small cavity and is solvent exposed (Fig. $\mathrm{S} 2$, left). The sulfane sulfur in the trisulfide points inward, sits at the apex of a $109^{\circ} \mathrm{S}-\mathrm{S}-\mathrm{S}$ angle, and is almost equidistant from the C4a atom in FAD as the S $\gamma$ of Cys-201. The $4.9 \AA$ (sulfane sulfur) and $4.2 \AA$ ( $\mathrm{S} \gamma$ of Cys-201) distances to $\mathrm{C} 4 \mathrm{a}$ in FAD in the representative structure from the most populated cluster in solution (Fig. 8A, left), are comparable to the 4.3 and $3.3 \AA$ distances observed in the trisulfide-containing crystal structure of $\mathrm{SQR}-\mathrm{CoQ}_{1}+$ sulfide (22). Inspection of the distribution of values for $\mathrm{S}_{\mathrm{C} 201}-\mathrm{C} 4 \mathrm{a}_{\mathrm{FAD}}$ and for the sulfane sulfur-C4a $\mathrm{a}_{\mathrm{FAD}}$ distances along the simulation (Fig. $8 \mathrm{~B}$, left) reveal broader histograms compared to the disulfide ones and a $0.5 \AA$ difference between the maxima.

The atomic charges calculated for the sulfur atoms in Cys-201, Cys-379 and the sulfane sulfur in the trisulfide, reveal a slightly electropositive reaction zone, particularly over Cys-379 $(+0.112$ atomic units Table S1), favoring attack of the negatively charged sulfide anion. On the other hand, the local softness for gaining electron density reveals that the $\mathrm{S} \gamma$ of Cys-379 and the sulfane sulfur are similar and significantly more reactive than the $\mathrm{S} \gamma$ of Cys-201.

Density Functional Theory (DFT) in a Polarized Continuum Model (PCM) characterization of the sulfide addition step-To gain further insights into the specific reactivities of the electrophilic sulfurs in a disulfide (Cys-201) versus a trisulfide (Cys-379) cofactor, we modeled the detailed mechanism using a DFT/PCM level of theory as described under Experimental Procedures. The $\mathrm{S}_{\mathrm{N}} 2$ mechanism involved the attack of a sulfide anion on the trisulfide or a hypothetical disulfide, with a persulfide or a thiolate anion, respectively serving as the leaving group (Fig. 8C). The transition states are quasi-linear, late, and quite synchronic, both in terms of heavy atom reorganization (HAR) and CT complex, with HAR/CT complex being more advanced in the disulfide compared to the trisulfide, as evidenced by the Wiberg Bond indices (WBI) and natural population analysis (NPA) charges (Tables S2-S5). The computed free energy barriers for the reaction of sulfide anion with the trisulfide versus disulfide cofactor in SQR are 9.0 and $15.3 \mathrm{kcal}$ $\mathrm{mol}^{-1}$ at $25{ }^{\circ} \mathrm{C}$ and $1 \mathrm{~atm}$, respectively. These results provide strong supporting evidence for the significantly greater reactivity of the trisulfide over the disulfide, accounting for much of the $10^{7}$-fold difference in the second order rate constant for the reaction of sulfide anion with SQR versus with a disulfide in solution [23]. The structure of the resulting CT product complex (Fig. S4) confirms completion of the $\mathrm{S}_{\mathrm{N}} 2$ reaction, and that the persulfide (or thiolate) can proceed to the next step in the reaction mechanism, concentrating excess negative charge at the ${ }^{201} \mathrm{Cys}_{-} \mathrm{SS}^{-}$or $\left({ }^{379} \mathrm{Cys}^{-} \mathrm{S}^{-}\right)$.

\section{Discussion}

Members of the flavoprotein disulfide reductase superfamily have a signature two redox cofactor active site constellation. While the flavin is common to all members, the second cofactor can be a cysteine disulfide, which is the most common theme in the superfamily, a cysteine sulfenic acid, or a mixed disulfide (e.g. Cys-S-S-CoA) (13). Recently, a fourth variation on the redox active cysteine cofactor theme, i.e., a trisulfide, was discovered in human SQR $(22,23)$.

Examples of cysteine trisulfides in proteins are rare. They have been observed primarily as artifacts in recombinant human growth hormone preparations (29-32). Trisulfide intermediates have been postulated as catalytic intermediates in dissimilatory sulfite reduction in bacteria (33) and in the SQR-catalyzed polysulfide formation in Aquifex aeolicus (18). The role of the cysteine trisulfide in human SQR is controversial $(22,23)$. Initially, it was proposed to result from a dead-end reaction with sulfide under anaerobic conditions in the absence of a sulfur acceptor (14). In this model, the active site cysteine disulfide in SQR would be regenerated via a chemically unusual mechanism that necessitates the elimination of S $\gamma$ of Cys-379 as an oxidized product, and replaces it with the sulfur atom derived from the trisulfide bridge (22). In addition to the unusual chemistry, the mechanism would require a significant conformational change to shorten the $\sim 3.5 \AA$ distance between the $\mathrm{S} \gamma$ of Cys-379 and Cys-201 to allow formation of a 
cysteine disulfide. Recent biochemical data from our laboratory have however, indicated that the trisulfide in SQR likely represents the active form of the enzyme (23). In this study, we disassembled and then reassembled the active site trisulfide by cyanolysis followed by sulfuration, and demonstrated that these processes led to the restoration of SQR activity.

The presence of the cysteine trisulfide in SQR was previously confirmed biochemically via cold cyanolysis (23), which extracts the bridging sulfur as thiocyanate (34). During the cyanolysis reaction, we had observed a rapid color change from yellow to blue, followed by a slow reversion to yellow, indicating the transient formation of a cyanide-induced CT complex followed by its decay. In the current study, this mechanism was supported by spectral and kinetic analyses, which demonstrate that cyanide, acting as a nucleophile, adds into the cysteine trisulfide (Fig. 2). We propose that cyanide attacks at the solventaccessible Cys-379, forming a ${ }^{379} \mathrm{Cys}-\mathrm{S}-\mathrm{C} \equiv \mathrm{N}$ organic thiocyanate and a ${ }^{201} \mathrm{Cys}-\mathrm{SS}^{-}$persulfide-to-FAD CT complex (Fig. 9, 2). The intense CT complex is similar to those seen with alternative nucleophiles such as sulfite or methanethiol adding to human SQR $(24,28)$ and also resembles the CT complex induced by coenzyme A persulfide in short-chain acyl-CoA dehydrogenase (23). The off-rate constant $\left(k_{\text {off }}=3.7 \pm 0.6\right.$ $\mathrm{s}^{-1}$ at $4{ }^{\circ} \mathrm{C}$ ) indicates that cyanide-induced CT complex formation is reversible, and that cyanide dissociation regenerates the trisulfide. Notably, cyanide can also act as a sulfur acceptor in the SQR reaction forming thiocyanate (14), and can thus contribute to the sulfide-mediated FAD reduction when both sulfide and cyanide are present. The ability of cyanide treated SQR to support catalysis indicated that the oxidation status of the active site cysteines was preserved in cyanolyzed enzyme.

The crystal structure of SQR provided a clue as to how the redox state of the cysteines is maintained upon cyanide treatment by revealing a bridging ${ }^{379} \mathrm{Cys} N-\left({ }^{201} \mathrm{Cys}\right.$-disulfanyl)-methanimido thioate intermediate (Fig. 7). We propose that this intermediate is formed by attack of the ${ }^{201}{ }^{2}$ ys-SS$^{-}$persulfide on the ${ }^{379} \mathrm{Cys}-\mathrm{S}-\mathrm{C} \equiv \mathrm{N}$ thiocyanate (Fig. 9, 3). While the bridging $N$-(disulfanyl)-methanimido thioate intermediate is stabilized in crystallo, it is susceptible to attack by a second equivalent of cyanide (Fig. 9, 4 ), leading to thiocyanate elimination, which was detected by the cold cyanolysis reaction. The resulting ${ }^{201} \mathrm{Cys}-\mathrm{S}-\mathrm{N}=\mathrm{CH}-\mathrm{S}-{ }^{379} \mathrm{Cys}$ intermediate (Fig. 9, 5) preserves the redox state of the active site cysteines. It does not however, support generation of a sulfite-induced CT complex (Fig. 3C), or FAD reduction in the presence of dithiols (Fig. 6 C, D), and it destabilizes SQR (Fig. 5). The absorption spectrum of FAD in the presence of this intermediate is subtly different from that in the native enzyme with the $450 \mathrm{~nm}$ peak blue shifted to $447 \mathrm{~nm}$, and the $380 \mathrm{~nm}$ peak being better resolved.

We propose that the trisulfide is rebuilt by the nucleophilic attack of sulfide on the S $\gamma$ of Cys-201, leading to a CT complex and then, to the resting enzyme (Fig. 9, $5 \rightarrow 2 \rightarrow 1$ ). We attribute the lag phase that was seen by stopped flow spectroscopy when sulfide was mixed with cyanide treated versus untreated SQR, to the time needed to rebuild the active enzyme trisulfide (Fig. 4A, B). Once rebuilt, the enzyme cycles through multiple catalytic turnovers and a difference in specific activities was not seen in cyanide treated versus untreated SQR under steady-state assay conditions.

The difference in the active site configurations of untreated versus cyanide treated SQR was further demonstrated by their differential reactivity to the dithiols DTT and DHLA. Both dithiols can substitute for sulfide in the oxidative half reaction, transferring electrons to FAD to form $\mathrm{FADH}_{2}$ (Fig. 6). Neither DTT nor DHLA reduced FAD in cyanide pretreated SQR, supporting the proposed mechanism (Fig. 6B).

Computational QM/MM and QM modeling provide strong evidence for the catalytic relevance of the trisulfide versus the disulfide form of the cofactor in SQR (Fig. 8). Based on accessibility, electrostatics and local softness considerations, the combination of MD simulations and QM/MM modeling predicted that Cys-379 in the trisulfide is the electrophilic target in the first step of SQR mechanism. Based on DFT/PCM modeling of the first step in the reaction mechanism, i.e. the attack by a sulfide anion, it was estimated that the trisulfide configuration affords an $\sim 10^{5}$-fold rate enhancement over a disulfide cofactor in the active site of SQR.

A significant question raised by the discovery of the trisulfide in SQR is how is the cofactor built? Minimally, one of two mechanisms can be considered. In the first, both cysteines are oxidized (e.g. to a sulfenic acid) followed by the attack of a sulfide anion to form a persulfide (e.g. on the solvent accessible 361 Cys-379), setting up trisulfide formation. In the second mechanism, both cysteines are persulfidated, an 


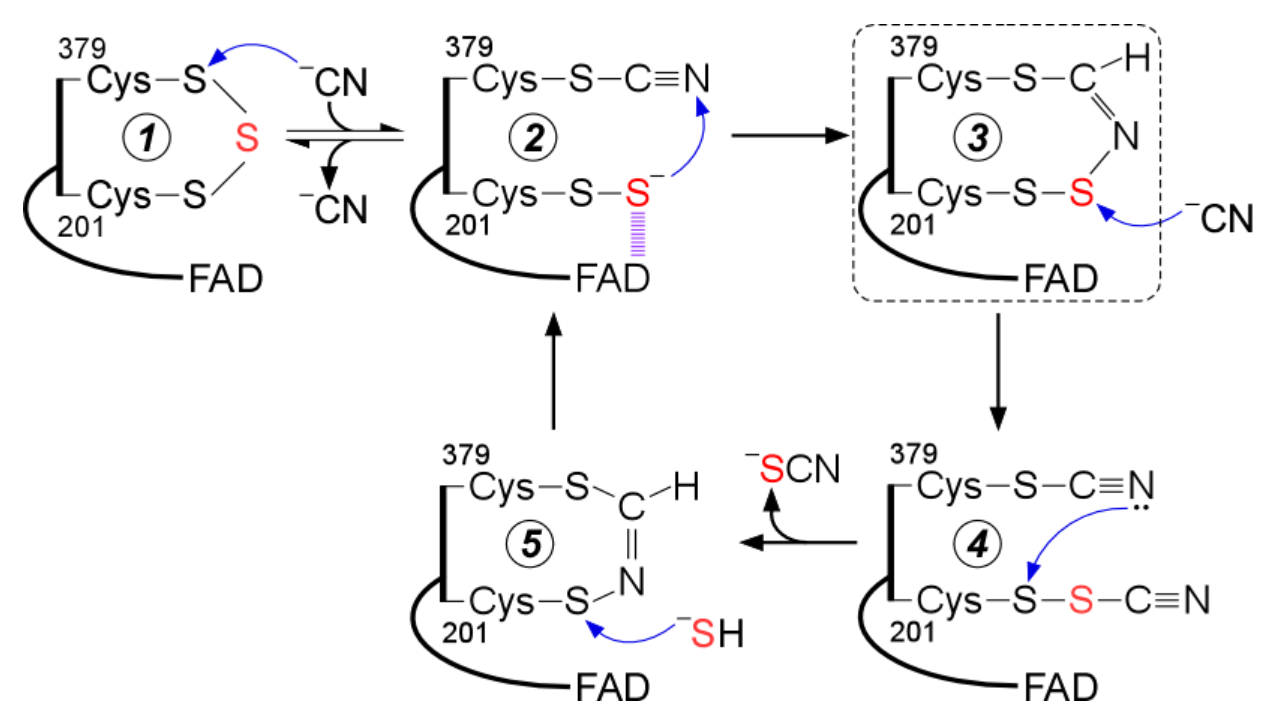

Figure 9. Proposed mechanism for cyanolysis and cysteine trisulfide rebuilding in SQR. Cyanide adds into the resting cysteine trisulfide (1) to generate a ${ }^{379} \mathrm{Cys}-\mathrm{S}-\mathrm{CN}$ organic thiocyanate while the bridging sulfur is retained in the ${ }^{201} \mathrm{Cys}^{-\mathrm{SS}^{-}}$persulfide that participates in a CT complex with FAD (2). Conversion to the ${ }^{379} \mathrm{Cys} N-\left({ }^{201}\right.$ Cys-disulfanyl)-methanimido thioate intermediate (3) leads to loss of the CT complex. Addition by a second cyanide at the sulfane sulfur of Cys-201 leads to intermediate (4), which can cyclize and eliminate thiocyanate (5), completing the cyanolysis reaction. Addition of sulfide to the S $\gamma$ of Cys-201 in the ${ }^{379} \mathrm{Cys} N$ $\left({ }^{201}\right.$ Cys-sulfanyl)-methanimido thioate intermediate (5) regenerates the CT complex (2). Elimination of cyanide regenerates the resting trisulfide form of the enzyme. The bridging sulfur of the cysteine trisulfide is labeled in red. The dashed box highlights the intermediate observed in the crystal structure.

oxidative cysteine modification that has been detected in many proteins (35). Low molecular weight persulfides (e.g. cysteine persulfide) could lead to formation of the bis-persulfide form of SQR from which the trisulfide could be built. Low molecular weight persulfides can be synthesized by all three $\mathrm{H}_{2} \mathrm{~S}$ generating enzymes $(6,7,36,37)$, and a potential role for these reactive sulfur species in signaling has been suggested (38). Alternatively, generation of the trisulfide could be catalyzed; candidate human sulfur transferases include rhodanese (39), mercaptopyruvate sulfurtransferase (6) and TSTD1 (40).

In summary, we have demonstrated that the cysteine trisulfide in human SQR can be reversibly dismantled and reassembled. The trisulfide not only contributes to a significant rate enhancement over a disulfide for the nucleophilic addition of sulfide, but also stabilizes the enzyme. Studies are underway in our laboratory to investigate whether assembly of the trisulfide is enzyme catalyzed.

\section{Materials and Methods}

Materials - The following reagents were purchased from Millipore Sigma: $\mathrm{CoQ}_{1}, \mathrm{n}$-dodecyl- $\beta$-D-maltoside (DDM), potassium cyanide, sodium sulfide nonahydrate, and sodium sulfite. The phospholipids, DHPC (1,2-diheptanoyl-sn-glycero-3-phosphocholine) and POPC, were purchased from Avanti Polar Lipids (Alabaster, AL). DHLA was purchased from Cayman Chemical Company (Ann Arbor, MI).

Preparation of human SQR-Human SQR was purified as detergent-solubilized recombinant enzyme as described previously (15). Human SQR used for crystallization was purified in an identical procedure as described previously (15), except that DHPC $(0.03 \% \mathrm{w} / \mathrm{v})$ was substituted with DDM $(0.05 \% \mathrm{w} / \mathrm{v})$ as the solubilizing detergent (23).

SQR spectral analyses and activity assays-The absorption spectra of SQR were recorded on a temperature-controlled Shimadzu UV-2600 spectrophotometer in Buffer A (50 mM Tris, pH 8.0, containing $300 \mathrm{mM} \mathrm{NaCl}$ and $0.03 \%$ DHPC). The concentration of SQR used in the spectral assays was estimated by the absorbance of the FAD cofactor, using an extinction coefficient of $11,500 \mathrm{M}^{-1} \mathrm{~cm}^{-1}$ at $450 \mathrm{~nm}$ (14). SQR activity was estimated by the rate of $\mathrm{CoQ}_{1}$ reduction $\left(\Delta \varepsilon_{\text {ox-red }}=12,000 \mathrm{M}^{-1} \mathrm{~cm}^{-1}\right)$ at $25{ }^{\circ} \mathrm{C}$ as described previously (15), using sulfite $(800 \mu \mathrm{M})$ as the sulfur acceptor. 
Stopped flow spectroscopy-All stopped flow experiments were conducted at $4{ }^{\circ} \mathrm{C}$ on a SF-DX2 double mixing stopped-flow system from Hi-Tech Scientific, equipped with a photodiode array detector (300-700 $\mathrm{nm}$ range). The concentrations reported in the figure legends for stopped flow experiments are before 1:1 $(\mathrm{v} / \mathrm{v})$ mixing.

Detection of sulfane sulfur in SQR-SQR was assayed for sulfane sulfur using the cold cyanolysis method as described previously (23). The data for mol sulfane sulfur per mol SQR monomer are presented as the mean $\pm \mathrm{SD}$ of three independent preparations of SQR.

Crystallization of $S Q R-C o Q_{1}$ with cyanide-SQR-CoQ ${ }_{1}$ crystals were grown at $20{ }^{\circ} \mathrm{C}$ by the hanging drop vapor diffusion method using solubilized human SQR $\left(17.4 \mathrm{mg} \mathrm{mL}^{-1}\right)$ in $50 \mathrm{mM}$ Tris- $\mathrm{HCl} \mathrm{pH} 8.0$, containing $\mathrm{NaCl}(300 \mathrm{mM})$ and n-dodecyl $\beta$-D-maltoside $(0.05 \% \mathrm{w} / \mathrm{v})$ supplemented with $\mathrm{CoQ}_{1}(5 \mathrm{mM}$, in $100 \%$ DMSO). The SQR solution was then mixed 1:1 (v/v) with the reservoir solution composed of 200 $\mathrm{mM}$ ammonium tartrate dibasic, $\mathrm{pH} 6.6$, and PEG $3350(20 \% \mathrm{w} / \mathrm{v})$, yielding a final $\mathrm{CoQ}_{1}$ concentration of $2.5 \mathrm{mM}$. The resulting SQR-CoQ ${ }_{1}$ crystals were soaked with potassium cyanide $(1.25 \mathrm{mM})$ for 40 min, followed by cryoprotection in the aforementioned reservoir solution supplemented with glycerol $(35 \% \mathrm{v} / \mathrm{v})$ before freezing in liquid nitrogen.

Thermal denaturation assays-The thermal stabilities of untreated SQR, and cyanide pre-treated SQR before and after sulfide treatment, were assessed using $300 \mu \mathrm{L}$ of enzyme $(5 \mu \mathrm{M})$ in a quartz cuvette, housed in a temperature-controlled Shimadzu UV-2600 spectrophotometer. SQR was allowed to equilibrate at 20 ${ }^{\circ} \mathrm{C}$ for $2 \mathrm{~min}$ before initiating the assay by increasing the temperature by $1{ }^{\circ} \mathrm{C} \mathrm{min}^{-1}$. Thermal denaturation was monitored by the increase in absorbance at $600 \mathrm{~nm}$.

$X$-ray data collection and structure determination-Diffraction data for SQR-CoQ $1+$ cyanide crystal was collected at the LS-CAT beamline 21-ID-D (Advanced Photon Source, Argonne National Laboratory) at $1.12723 \AA$ wavelength. The diffraction images were processed using HKL2000 (41). The molecular replacement solution for SQR-CoQ ${ }_{1}+$ cyanide was determined using SQR-CoQ 1 (PDB ID: 6OIB) as a search model. The final structures were completed using alternate cycles of manual fitting in Coot (42) and refinement in REFMAC5 (43). The stereochemical quality of the final models was assessed using MolProbity (44).

$M D$ simulations of $S Q R$-The crystal structure of human $\mathrm{SQR}-\mathrm{CoQ}_{1}+$ sulfide in the trisulfide state complexed with FAD (PDB: 6OI6, monomer A, 2.56 Á resolution) was used as a starting point (23). CoQ 1 was manually docked by superimposing another structure of SQR-CoQ 1 (PDB: 6OIB, monomer A, $2.03 \AA$ resolution). Two systems were simulated: SQR in a Cys-201-Cys-379 disulfide state (SQR-SS) and in the trisulfide state (SQR-SSS). Protonation and tautomers of titratable residues, and missing hydrogen atoms, were added with the ProToss utility (45). Systems were solvated with a periodic truncated octahedral box of TIP3P water extended up to $12 \AA$ around solute, then neutralized with six $\mathrm{Cl}^{-}$ions with the leap utility of AmberTools17 (46). Both were then minimized, heated to $310 \mathrm{~K}(500 \mathrm{ps}$, NVT), and equilibrated at 1 atm (1 ns, NPT) prior to conducting the simulations (600 ns, NPT). Minimization and simulation was carried out using the pmemd.cuda module of AMBER 16 (46). For describing the protein, the AMBER $f f 14 S B$ force field was used for standard residues, whereas the gaff force field was used for FAD and CoQ 1 ligands, along with RESP charges (47). The central sulfur atom of the trisulfide moiety was treated as a separate residue with zero charge. All parameters for describing the trisulfide moiety were present in the ff1 $14 S B$ force field, except for the S-S-S bonds, which were taken from the gaff force field. An 8.0 A cutoff was used for treating direct non-bonding interactions, and long-range interactions were treated with the Particle Mesh Ewald (PME) method (48). For MD simulations, temperature and pressure (in NPT simulations) were controlled by means of the Langevin thermostat (49) and the Monte Carlo barostat (50), respectively. Distances involving hydrogen atoms were constrained with SHAKE (51) and a time integration step of 2 femtoseconds was used. Harmonic restraints of $10 \mathrm{kcal} \mathrm{mol}^{-1} \AA^{-2}$ were applied to a water-bridged hydrogen bond observed in SQR crystal structures (23) between the protonated amino group of the Lys-207 side chain and the N5 atom in FAD. Trajectory processing and analysis was done with the cpptraj module of AmberTools17 (46). Convergence of simulations was monitored following the $\mathrm{C} \alpha$ RMSD (see Fig. S1). In order to extract representative structures from the MD 600 ns trajectories, clustering 
analysis (5 clusters) was performed for each system, using a hierarchical-agglomerative algorithm with cpptraj (46).

Reactivity descriptors from $Q M / M M$ calculations on $S Q R$-Descriptors of intrinsic reactivity derived from the electronic structure of Cys-379 and Cys-201 were calculated at the QM/MM level in the framework of a conceptual DFT (52) at the M06-2X-D3/6-31+G(d,p) level of theory (53-55) combined to a classical description of the enzyme using the aforementioned force fields. Global softness $(S)$ and electrophilic Fukui function condensed to the $\mathrm{S} \gamma$ atom $\left(f_{S \gamma}^{+}\right)$were calculated according to equations (1) and (2):

$$
S=\frac{1}{I P-E A}
$$

where $I P$ and $\mathrm{E} A$ respectively represent the ionization potential and electron affinity of the system of interest, determined using the vertical $\triangle \mathrm{SCF}$ (self-consistent field) approximation (56), and

$$
f_{\mathrm{S} \gamma}^{+}=q_{\mathrm{S} \gamma}(N)-q_{\mathrm{S} \gamma}(N+1)
$$

where $q_{\mathrm{S} \gamma}(N)$ and $q_{\mathrm{S} \gamma}(N+1)$ represent the atomic charge on the S $\gamma$ atoms of Cys-379/Cys-201, calculated using a Natural Population Analysis (57), both in the system of reference bearing $\mathrm{N}$ electrons and after addition of one extra electron. The atomic electrophilic softness $\left(s_{\mathrm{S} \gamma}^{+}\right.$, calculated as $S$ times $\left.f_{\mathrm{S} \gamma}^{+}\right)$is a local descriptor that can be used to compare $\mathrm{S} \gamma$ intrinsic reactivity in Cys-379 and Cys-201 across SQR-SS and SQR-SSS. The electronic structure of each macromolecular system was thus obtained through single-point calculations performed on representative structures extracted from MD simulations using the additive QM/MM scheme implemented in AMBER16 (46) interfaced with Gaussian 09 Rev. D.01 (58) with a QM region comprising ${ }^{379} \mathrm{Cys}-\mathrm{CH}_{2}-\mathrm{S}(\mathrm{S}) \mathrm{S}-\mathrm{CH}_{2}{ }^{201} \mathrm{Cys}$.

DFT-PCM modeling of reaction mechanisms and barriers for sulfide nucleophilic attack- The mechanism of the reaction of the sulfide anion, manually docked and oriented as guided by our previous models of similar reactions (59), was characterized at the M06-2X-D3/6-31+G(d,p)-PCM level of theory, previously validated by us to model reactions of the sulfide anion in a similar system (59). A simplified representation of the catalytic disulfide/trisulfide and FAD at the active site of SQR was used including $\mathrm{CH}_{3} \mathrm{SSSCH}_{3} / \mathrm{CH}_{3} \mathrm{SSCH}_{3}$ and the flavin. The structures of each reactant complex, transition and product complex were fully optimized and verified by the inspection of the eigenvalues of the Hessian matrix at the same level. Thermochemical corrections at $298 \mathrm{~K}$ and $1 \mathrm{~atm}$ were calculated under usual approximations of statistical thermodynamics (rigid rotor, harmonic frequencies) as implemented in Gaussian 09 Rev. D01 (58). The effects exerted by the bulk protein on the active site structure along the reaction and reaction barrier were introduced using the IEF-PCM continuum model (60) with a dielectric constant $\varepsilon=10.125$. The reactive systems were placed in a molecular shaped cavity constructed using Bondi's radii (61) and including non-electrostatic (cavitation, repulsion and dispersion) contributions. In order to connect transition states with reactant complexes and product complexes, we calculated the IRC reaction path (62) using the HPC algorithm (63).

\section{Acknowledgements}

We thank Drs. David P. Ballou and Markus Ruetz for insightful discussions on the cysteine trisulfide cyanolysis and rebuilding mechanisms. MD simulations were carried out using Uruguayan supercomputer resources from ClusterUY (https://cluster.uy). Continuous support from PEDEClBA-Uruguay is gratefully acknowledged by JB and ELC who are active members of the Uruguayan National Research System (SNIANII).

\section{Author Disclosure Statement}

No competing financial interest exists. 


\section{Author contributions}

498 A.P.L. designed and performed the kinetic and spectroscopic experiments. S.M. determined the crystal 499 structure, which was analyzed together with U.S.C. J.B. performed the computational modeling and MD 500 simulations, which were analyzed together with E.L.C. R.B. helped conceive the experiments, analyzed the 501 data and co-wrote the manuscript with A.P.L. and S.M. with the exception of the computational sections 502 that were co-written by J.B. and E.L.C. All authors approved the final version of the manuscript. 


\section{References}

1. Kimura, H. (2010) Hydrogen sulfide: from brain to gut. Antioxid Redox Signal 12, 1111-1123

2. Kabil, O., and Banerjee, R. (2010) Redox biochemistry of hydrogen sulfide. J Biol Chem $\mathbf{2 8 5}$, 21903-21907

3. Filipovic, M. R., Zivanovic, J., Alvarez, B., and Banerjee, R. (2018) Chemical Biology of H2S Signaling through Persulfidation. Chem Rev 118, 1253-1337

4. Singh, S., and Banerjee, R. (2011) PLP-dependent H(2)S biogenesis. Biochim Biophys Acta 1814, 1518-1527

5. Chiku, T., Padovani, D., Zhu, W., Singh, S., Vitvitsky, V., and Banerjee, R. (2009) H2S biogenesis by human cystathionine gamma-lyase leads to the novel sulfur metabolites lanthionine and homolanthionine and is responsive to the grade of hyperhomocysteinemia. J Biol Chem 284, 11601-11612

6. Yadav, P. K., Yamada, K., Chiku, T., Koutmos, M., and Banerjee, R. (2013) Structure and kinetic analysis of $\mathrm{H} 2 \mathrm{~S}$ production by human mercaptopyruvate sulfurtransferase. J Biol Chem 288, 20002-20013

7. Yadav, P. K., Vitvitsky, V., Carballal, S., Seravalli, J., and Banerjee, R. (2020) Thioredoxin regulates human mercaptopyruvate sulfurtransferase at physiologically-relevant concentrations. $J$ Biol Chem 295, 6299-6311

8. Furne, J., Saeed, A., and Levitt, M. D. (2008) Whole tissue hydrogen sulfide concentrations are orders of magnitude lower than presently accepted values. Am J Physiol Regul Integr Comp Physiol 295, R1479-1485

9. Levitt, M. D., Abdel-Rehim, M. S., and Furne, J. (2011) Free and acid-labile hydrogen sulfide concentrations in mouse tissues: anomalously high free hydrogen sulfide in aortic tissue. Antioxid Redox Signal 15, 373-378

10. Vitvitsky, V., Kabil, O., and Banerjee, R. (2012) High turnover rates for hydrogen sulfide allow for rapid regulation of its tissue concentrations. Antioxid Redox Signal 17, 22-31

11. Bouillaud, F., and Blachier, F. (2011) Mitochondria and sulfide: a very old story of poisoning, feeding, and signaling? Antioxid Redox Signal 15, 379-391

12. Hildebrandt, T. M., and Grieshaber, M. K. (2008) Three enzymatic activities catalyze the oxidation of sulfide to thiosulfate in mammalian and invertebrate mitochondria. FEBS J 275, 3352-3361

13. Argyrou, A., and Blanchard, J. S. (2004) Flavoprotein disulfide reductases: advances in chemistry and function. Prog Nucleic Acid Res Mol Biol 78, 89-142

14. Jackson, M. R., Melideo, S. L., and Jorns, M. S. (2012) Human sulfide:quinone oxidoreductase catalyzes the first step in hydrogen sulfide metabolism and produces a sulfane sulfur metabolite. Biochemistry 51, 6804-6815

15. Libiad, M., Yadav, P. K., Vitvitsky, V., Martinov, M., and Banerjee, R. (2014) Organization of the human mitochondrial hydrogen sulfide oxidation pathway. J Biol Chem 289, 30901-30910

16. Landry, A. P., Ballou, D. P., and Banerjee, R. (2017) $\mathrm{H}_{2} \mathrm{~S}$ oxidation by nanodisc-embedded human sulfide quinone oxidoreductase. J Biol Chem 292, 11641-11649

17. Friederich, M. W., Elias, A. F., Kuster, A., Laugwitz, L., Larson, A. A., Landry, A. P., EllwoodDigel, L., Mirsky, D. M., Dimmock, D., and Haven, J. (2020) Pathogenic variants in SQOR encoding sulfide: quinone oxidoreductase are a potentially treatable cause of Leigh disease. $J$ Inherited Metab Dis, 10.1002/jimd.12232

18. Marcia, M., Ermler, U., Peng, G., and Michel, H. (2009) The structure of Aquifex aeolicus sulfide:quinone oxidoreductase, a basis to understand sulfide detoxification and respiration. Proc Natl Acad Sci U S A 106, 9625-9630

19. Brito, J. A., Sousa, F. L., Stelter, M., Bandeiras, T. M., Vonrhein, C., Teixeira, M., Pereira, M. M., and Archer, M. (2009) Structural and functional insights into sulfide:quinone oxidoreductase. Biochemistry 48, 5613-5622 
20. Cherney, M. M., Zhang, Y., Solomonson, M., Weiner, J. H., and James, M. N. (2010) Crystal structure of sulfide:quinone oxidoreductase from Acidithiobacillus ferrooxidans: insights into sulfidotrophic respiration and detoxification. J Mol Biol 398, 292-305

21. Chen, Z. W., Koh, M., Van Driessche, G., Van Beeumen, J. J., Bartsch, R. G., Meyer, T. E., Cusanovich, M. A., and Mathews, F. S. (1994) The structure of flavocytochrome c sulfide dehydrogenase from a purple phototrophic bacterium. Science 266, 430-432

22. Jackson, M. R., Loll, P. J., and Jorns, M. S. (2019) X-Ray Structure of Human Sulfide:Quinone Oxidoreductase: Insights into the Mechanism of Mitochondrial Hydrogen Sulfide Oxidation. Structure 27, 794-805 e794

23. Landry, A. P., Moon, S., Kim, H., Yadav, P. K., Guha, A., Cho, U. S., and Banerjee, R. (2019) A Catalytic Trisulfide in Human Sulfide Quinone Oxidoreductase Catalyzes Coenzyme A Persulfide Synthesis and Inhibits Butyrate Oxidation. Cell Chem Biol 26, 1515-1525 e1514

24. Mishanina, T. V., Yadav, P. K., Ballou, D. P., and Banerjee, R. (2015) Transient Kinetic Analysis of Hydrogen Sulfide Oxidation Catalyzed by Human Sulfide Quinone Oxidoreductase. J Biol Chem 290, 25072-25080

25. Goubern, M., Andriamihaja, M., Nubel, T., Blachier, F., and Bouillaud, F. (2007) Sulfide, the first inorganic substrate for human cells. FASEB J 21, 1699-1706

26. Benchoam, D., Cuevasanta, E., Moller, M. N., and Alvarez, B. (2020) Persulfides, at the crossroads between hydrogen sulfide and thiols. Essays Biochem 64, 155-168

27. Cuevasanta, E., Lange, M., Bonanata, J., Coitino, E. L., Ferrer-Sueta, G., Filipovic, M. R., and Alvarez, B. (2015) Reaction of Hydrogen Sulfide with Disulfide and Sulfenic Acid to Form the Strongly Nucleophilic Persulfide. J Biol Chem 290, 26866-26880

28. Landry, A. P., Ballou, D. P., and Banerjee, R. (2018) Modulation of Catalytic Promiscuity during Hydrogen Sulfide Oxidation. ACS Chem Biol 13, 1651-1658

29. Jespersen, A. M., Christensen, T., Klausen, N. K., Nielsen, F., and Sorensen, H. H. (1994) Characterisation of a trisulphide derivative of biosynthetic human growth hormone produced in Escherichia coli. Eur J Biochem 219, 365-373

30. Thomsen, M. K., Hansen, B. S., Nilsson, P., Nowak, J., Johansen, P. B., Thomsen, P. D., and Christiansen, J. (1994) Pharmacological characterization of a biosynthetic trisulfide-containing hydrophobic derivative of human growth hormone: comparison with standard $22 \mathrm{~K}$ growth hormone. Pharmacol Toxicol 74, 351-358

31. Andersson, C., Edlund, P. O., Gellerfors, P., Hansson, Y., Holmberg, E., Hult, C., Johansson, S., Kordel, J., Lundin, R., Mendel-Hartvig, I. B., Noren, B., Wehler, T., Widmalm, G., and Ohman, J. (1996) Isolation and characterization of a trisulfide variant of recombinant human growth hormone formed during expression in Escherichia coli. Int J Pept Protein Res 47, 311-321

32. Canova-Davis, E., Baldonado, I. P., Chloupek, R. C., Ling, V. T., Gehant, R., Olson, K., and Gillece-Castro, B. L. (1996) Confirmation by mass spectrometry of a trisulfide variant in methionyl human growth hormone biosynthesized in Escherichia coli. Anal Chem 68, 4044-4051

33. Santos, A. A., Venceslau, S. S., Grein, F., Leavitt, W. D., Dahl, C., Johnston, D. T., and Pereira, I. A. (2015) A protein trisulfide couples dissimilatory sulfate reduction to energy conservation. Science 350, 1541-1545

34. Wood, J. L. (1987) Sulfane sulfur. Methods Enzymol 143, 25-29

35. Gao, X. H., Krokowski, D., Guan, B. J., Bederman, I., Majumder, M., Parisien, M., Diatchenko, L., Kabil, O., Willard, B., Banerjee, R., Wang, B., Bebek, G., Evans, C. R., Fox, P. L., Gerson, S. L., Hoppel, C. L., Liu, M., Arvan, P., and Hatzoglou, M. (2015) Quantitative $\mathrm{H}_{2} \mathrm{~S}$-mediated protein sulfhydration reveals metabolic reprogramming during the integrated stress response. Elife 4, e10067

36. Ida, T., Sawa, T., Ihara, H., Tsuchiya, Y., Watanabe, Y., Kumagai, Y., Suematsu, M., Motohashi, H., Fujii, S., Matsunaga, T., Yamamoto, M., Ono, K., Devarie-Baez, N. O., Xian, M., Fukuto, J. M., and Akaike, T. (2014) Reactive cysteine persulfides and S-polythiolation regulate oxidative stress and redox signaling. Proc Natl Acad Sci U S A 111, 7606-7611 
604

605

606

607

608

609

610

611

612

613

614

615

616

617

618

619

620

621

622

623

624

625

626

627

628

629

630

631

632

633

634

635

636

637

638

639

640

641

642

643

644

645

646

647

648

649

650

651

652

653

37. Yadav, P. K., Martinov, M., Vitvitsky, V., Seravalli, J., Wedmann, R., Filipovic, M. R., and Banerjee, R. (2016) Biosynthesis and Reactivity of Cysteine Persulfides in Signaling. J Am Chem Soc 138, 289-299

38. Mishanina, T. V., Libiad, M., and Banerjee, R. (2015) Biogenesis of reactive sulfur species for signaling by hydrogen sulfide oxidation pathways. Nat Chem Biol 11, 457-464

39. Libiad, M., Sriraman, A., and Banerjee, R. (2015) Polymorphic Variants of Human Rhodanese Exhibit Differences in Thermal Stability and Sulfur Transfer Kinetics. J Biol Chem 290, 2357923588

40. Libiad, M., Motl, N., Akey, D. L., Sakamoto, N., Fearon, E. R., Smith, J. L., and Banerjee, R. (2018) Thiosulfate sulfurtransferase-like domain-containing 1 protein interacts with thioredoxin. $J$ Biol Chem 293, 2675-2686

41. Otwinowski, Z., and Minor, W. (1997) Processing of X-ray diffraction data collected in oscillation mode. Methods Enzymol 276, 307-326

42. Emsley, P., and Cowtan, K. (2004) Coot: model-building tools for molecular graphics. Acta Crystallogr D Biol Crystallogr 60, 2126-2132

43. Murshudov, G. N., Vagin, A. A., and Dodson, E. J. (1997) Refinement of macromolecular structures by the maximum-likelihood method. Acta Crystallogr D Biol Crystallogr 53, 240-255

44. Chen, V. B., Arendall, W. B., 3rd, Headd, J. J., Keedy, D. A., Immormino, R. M., Kapral, G. J., Murray, L. W., Richardson, J. S., and Richardson, D. C. (2010) MolProbity: all-atom structure validation for macromolecular crystallography. Acta Crystallogr D Biol Crystallogr 66, 12-21

45. Bietz, S., Urbaczek, S., Schulz, B., and Rarey, M. (2014) Protoss: a holistic approach to predict tautomers and protonation states in protein-ligand complexes. J Cheminform 6, 12

46. Case, D. A., Cerutti, D. S., Cheatham III, T. E., Darden, T. A., Duke, R. E., Giese, T. J., Gohlke, H., A.W., G., Greene, D., Homeyer, N., Izadi, S., Kovalenko, A., Lee, T. S., LeGrand, S., Li, P., Lin, C., Liu, J., Luchko, T., Luo, R., Mermelstein, D., Merz, K. M., Monard, G., Nguyen, H., Omelyan, I., Onufriev, A., Pan, F., Qi, R., Roe, D. R., Roitberg, A., Sagui, C., Simmerling, C. L., Botello-Smith, W. M., Swails, J., Walker, R. C., Wang, J., Wolf, R. M., Wu, X., Xiao, L., York, D. M., and Kollman, P. A. (2017) AMBER 2017. University of California, San Francisco.

47. Bayly, C. I., Cieplak, P., Cornell, W., and Kollman, P. A. (1993) A well-behaved electrostatic potential based method using charge restraints for deriving atomic charges: the RESP model. The Journal of Physical Chemistry 97, 10269-10280

48. Essmann, U., Perera, L., Berkowitz, M. L., Darden, T., Lee, H., and Pedersen, L. G. (1995) A smooth particle mesh Ewald method. The Journal of Chemical Physics 103, 8577-8593

49. Loncharich, R. J., Brooks, B. R., and Pastor, R. W. (1992) Langevin dynamics of peptides: the frictional dependence of isomerization rates of N-acetylalanyl-N'-methylamide. Biopolymers 32, 523-535

50. Åqvist, J., Wennerström, P., Nervall, M., Bjelic, S., and Brandsdal, B. O. (2004) Molecular dynamics simulations of water and biomolecules with a Monte Carlo constant pressure algorithm. Chem Phys Lett 384, 288-294

51. Ryckaert, J.-P., Ciccotti, G., and Berendsen, H. J. C. (1977) Numerical integration of the cartesian equations of motion of a system with constraints: molecular dynamics of n-alkanes. Journal of computational physics 23, 327-341

52. Geerlings, P., De Proft, F., and Langenaeker, W. (2003) Conceptual density functional theory. Chem Rev 103, 1793-1873

53. Francl, M. M., Pietro, W. J., Hehre, W. J., Binkley, J. S., Gordon, M. S., DeFrees, D. J., and Pople, J. A. (1982) Self-consistent molecular orbital methods. XXIII. A polarization-type basis set for second-row elements. The Journal of Chemical Physics 77, 3654-3665

54. Grimme, S., Antony, J., Ehrlich, S., and Krieg, H. (2010) A consistent and accurate ab initio parametrization of density functional dispersion correction (DFT-D) for the 94 elements H-Pu. $J$ Chem Phys 132, 154104 
654
55. Zhao, Y., and Truhlar, D. G. (2008) The M06 suite of density functionals for main group thermochemistry, thermochemical kinetics, noncovalent interactions, excited states, and transition elements: two new functionals and systematic testing of four M06-class functionals and 12 other functionals. Theor Chem Acc 120, 215-241

56. Jones, R. O., and Gunnarsson, O. (1989) The density functional formalism, its applications and prospects. Reviews of Modern Physics 61, 689

57. Foster, a. J. P., and Weinhold, F. (1980) Natural hybrid orbitals. J Am Chem Soc 102, 7211-7218

58. Frisch, M. J., Trucks, G. W., Schlegel, H. B., Scuseria, G. E., Robb, M. A., Cheeseman, J. R., Scalmani, G., Barone, V., Mennucci, B., Petersson, G. A., Nakatsuji, H., Caricato, M., Li, X., Hratchian, H. P., Izmaylov, A. F., Bloino, J., Zheng, G., Sonnenberg, J. L., Hada, M., Ehara, M., Toyota, K., Fukuda, R., Hasegawa, J., Ishida, M., Nakajima, T., Honda, Y., Kitao, O., Nakai, H., Vreven, T., Montgomery Jr., J. A., Peralta, J. E., Ogliaro, F., Bearpark, M. J., Heyd, J., Brothers, E. N., Kudin, K. N., Staroverov, V. N., Kobayashi, R., Normand, J., Raghavachari, K., Rendell, A. P., Burant, J. C., Iyengar, S. S., Tomasi, J., Cossi, M., Rega, N., Millam, N. J., Klene, M., Knox, J. E., Cross, J. B., Bakken, V., Adamo, C., Jaramillo, J., Gomperts, R., Stratmann, R. E., Yazyev, O., Austin, A. J., Cammi, R., Pomelli, C., Ochterski, J. W., Martin, R. L., Morokuma, K., Zakrzewski, V. G., Voth, G. A., Salvador, P., Dannenberg, J. J., Dapprich, S., Daniels, A. D., Farkas, Ö., Foresman, J. B., Ortiz, J. V., Cioslowski, J., and Fox, D. J. (2013) Gaussian 09 Revision D.01. Gaussian, Inc., Wallingford, CT.

59. Bonanata, J., and Coitino, E. L. (2019) Understanding the mechanism of H2S oxidation by flavindependent sulfide oxidases: a DFT/IEF-PCM study. J Mol Model 25, 308

60. Mennucci, B. (2012) Polarizable continuum model. Wiley Interdisciplinary Reviews: Computational Molecular Science 2, 386-404

61. Bondi, A. v. (1964) van der Waals volumes and radii. The Journal of physical chemistry $\mathbf{6 8}, 441-$ 451

62. Fukui, K. (1981) The path of chemical reactions-the IRC approach. Acc Chem Res 14, 363-368

63. Hratchian, H. P., and Schlegel, H. B. (2005) Using Hessian updating to increase the efficiency of a Hessian based predictor-corrector reaction path following method. J Chem Theory Comput 1, 6169 